\title{
SUPPLEMENTAL ARTICLE: BAYESIAN SHRINKAGE METHODS FOR PARTIALLY OBSERVED DATA WITH MANY PREDICTORS
}

\author{
By PHILIP S. BOONSTRA, BHRAMAR MUKHERJEE, AND JEREMY MG TAYLOR, \\ University of Michigan
}

\section{APPENDIX A: ENUMERATION OF GIBBS STEPS}

The complete-data log-likelihood, given in expression (4) in the manuscript, is

$$
\begin{aligned}
\ell_{C}=\ln \left[\boldsymbol{U}^{\mathrm{obs}}, \boldsymbol{U}^{\mathrm{mis}} \mid \boldsymbol{\phi}\right]= & \ln \left[\boldsymbol{y}_{\mathrm{A}} \mid \boldsymbol{x}_{\mathrm{A}}, \beta_{0}, \boldsymbol{\beta}, \sigma^{2}\right]+\ln \left[\boldsymbol{w}_{\mathrm{A}} \mid \boldsymbol{x}_{\mathrm{A}}, \psi, \nu, \tau^{2}\right]+\ln \left[\boldsymbol{x}_{\mathrm{A}} \mid \boldsymbol{\mu}_{\boldsymbol{X}}, \boldsymbol{\Sigma}_{\boldsymbol{X}}\right] \\
& +\ln \left[\boldsymbol{y}_{\mathrm{B}} \mid \boldsymbol{x}_{\mathrm{B}}, \beta_{0}, \boldsymbol{\beta}, \sigma^{2}\right]+\ln \left[\boldsymbol{w}_{\mathrm{B}} \mid \boldsymbol{x}_{\mathrm{B}}, \psi, \nu, \tau^{2}\right]+\ln \left[\boldsymbol{x}_{\mathrm{B}} \mid \boldsymbol{\mu}_{\boldsymbol{X}}, \boldsymbol{\Sigma}_{\boldsymbol{X}}\right] .
\end{aligned}
$$

For a choice of prior, $[\phi]$, the partial conditional distributions implied by the product of the likelihood and the prior, $\left[\boldsymbol{U}^{\text {obs }}, \boldsymbol{U}^{\text {mis }} \mid \boldsymbol{\phi}\right] \times[\phi]$, will yield the Gibbs steps. These are discussed in the order presented in the manuscript.

VANILLA The prior is given in expression (6) in the manuscript:

$$
[\boldsymbol{\phi}]=\left[\beta_{0}, \boldsymbol{\beta}, \sigma^{2}, \psi, \nu, \tau^{2}, \boldsymbol{\mu}_{\boldsymbol{X}}, \boldsymbol{\Sigma}_{\boldsymbol{X}}^{-1}\right] \propto\left(\sigma^{2} \tau^{2}\right)^{-1}\left|\boldsymbol{\Sigma}_{\boldsymbol{X}}^{-1}\right|^{(2 p-1) / 2} \exp \left\{-\frac{2 p-1}{2} \operatorname{Tr}\left(\operatorname{diag}\left(\hat{\operatorname{Var}}\left[\boldsymbol{x}_{\mathrm{A}}\right]\right) \boldsymbol{\Sigma}_{\boldsymbol{X}}^{-1}\right)\right\},
$$

where $\operatorname{diag}\left(\hat{\operatorname{Var}}\left[\boldsymbol{x}_{\mathrm{A}}\right]\right)$ is the diagonal part of the empirical covariance of $\boldsymbol{x}_{\mathrm{A}}$. This is a Jeffreys prior on each parameter except $\boldsymbol{\Sigma}_{\boldsymbol{X}}^{-1}$, and $\boldsymbol{\eta}$ (the vector of hyperparameters) is specified. Each Gibbs step is as follows: 


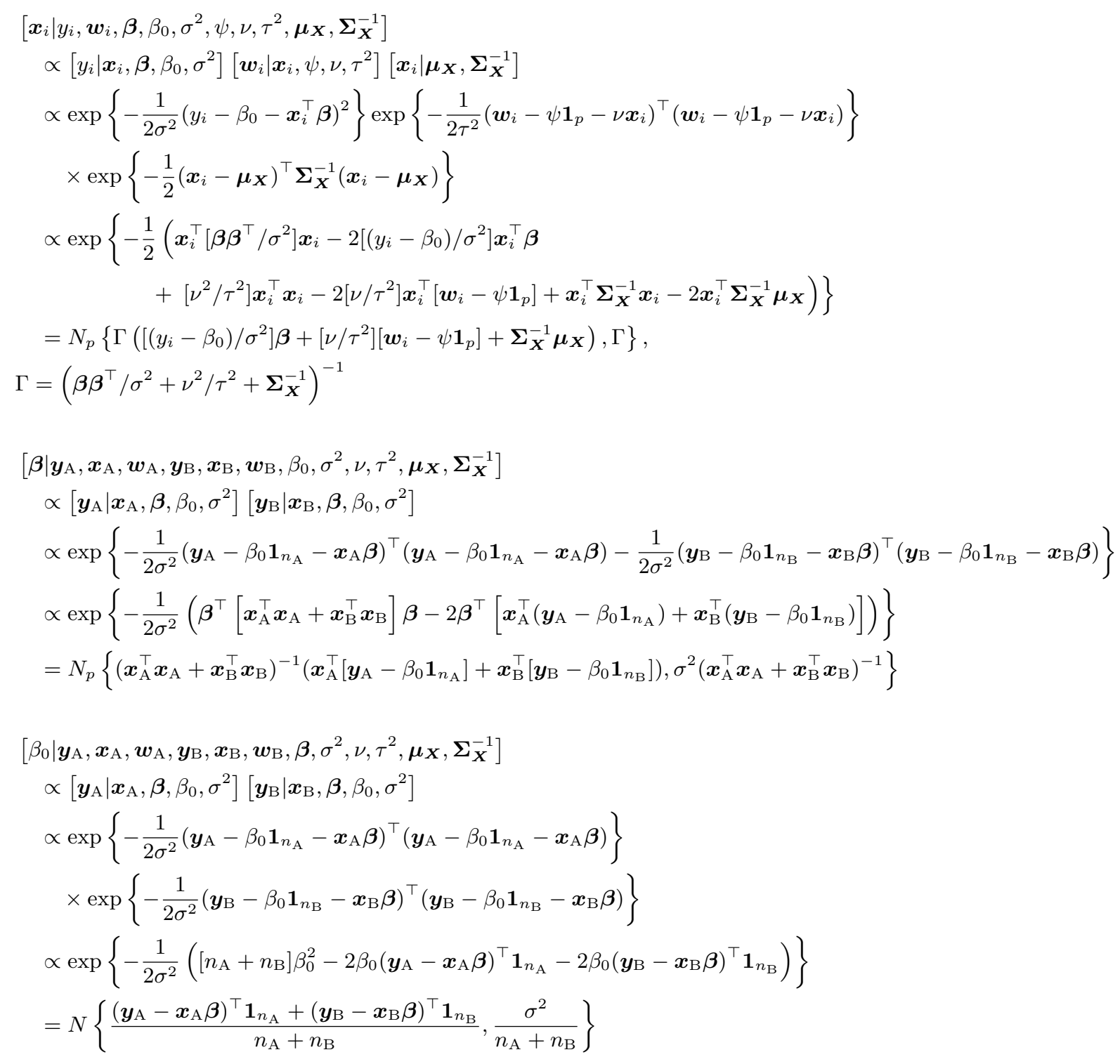




$$
\begin{aligned}
& {\left[\sigma^{2} \mid \boldsymbol{y}_{\mathrm{A}}, \boldsymbol{x}_{\mathrm{A}}, \boldsymbol{w}_{\mathrm{A}}, \boldsymbol{y}_{\mathrm{B}}, \boldsymbol{x}_{\mathrm{B}}, \boldsymbol{w}_{\mathrm{B}}, \boldsymbol{\beta}, \beta_{0}, \nu, \tau^{2}, \boldsymbol{\mu}_{\boldsymbol{X}}, \boldsymbol{\Sigma}_{\boldsymbol{X}}^{-1}\right]} \\
& \propto\left[\boldsymbol{y}_{\mathrm{A}} \mid \boldsymbol{x}_{\mathrm{A}}, \boldsymbol{\beta}, \beta_{0}, \sigma^{2}\right]\left[\boldsymbol{y}_{\mathrm{B}} \mid \boldsymbol{x}_{\mathrm{B}}, \boldsymbol{\beta}, \beta_{0}, \sigma^{2}\right] \\
& \propto\left(\sigma^{2}\right)^{-n_{\mathrm{A}} / 2} \exp \left\{-\frac{1}{2 \sigma^{2}}\left(\boldsymbol{y}_{\mathrm{A}}-\beta_{0} \mathbf{1}_{n_{\mathrm{A}}}-\boldsymbol{x}_{\mathrm{A}} \boldsymbol{\beta}\right)^{\top}\left(\boldsymbol{y}_{\mathrm{A}}-\beta_{0} \mathbf{1}_{n_{\mathrm{A}}}-\boldsymbol{x}_{\mathrm{A}} \boldsymbol{\beta}\right)\right\} \\
& \times\left(\sigma^{2}\right)^{-n_{\mathrm{B}} / 2} \exp \left\{-\frac{1}{2 \sigma^{2}}\left(\boldsymbol{y}_{\mathrm{B}}-\beta_{0} \mathbf{1}_{n_{\mathrm{B}}}-\boldsymbol{x}_{\mathrm{B}} \boldsymbol{\beta}\right)^{\top}\left(\boldsymbol{y}_{\mathrm{B}}-\beta_{0} \mathbf{1}_{n_{\mathrm{B}}}-\boldsymbol{x}_{\mathrm{B}} \boldsymbol{\beta}\right)\right\}\left(\sigma^{2}\right)^{-1} \\
& =I G\left\{\frac{1}{2}\left(n_{\mathrm{A}}+n_{\mathrm{B}}\right)\right. \text {, } \\
& \frac{1}{2}\left(\boldsymbol{y}_{\mathrm{A}}-\beta_{0} \mathbf{1}_{n_{\mathrm{A}}}-\boldsymbol{x}_{\mathrm{A}} \boldsymbol{\beta}\right)^{\top}\left(\boldsymbol{y}_{\mathrm{A}}-\beta_{0} \mathbf{1}_{n_{\mathrm{A}}}-\boldsymbol{x}_{\mathrm{A}} \boldsymbol{\beta}\right) \\
& \left.+\frac{1}{2}\left(\boldsymbol{y}_{\mathrm{B}}-\beta_{0} \mathbf{1}_{n_{\mathrm{B}}}-\boldsymbol{x}_{\mathrm{B}} \boldsymbol{\beta}\right)^{\top}\left(\boldsymbol{y}_{\mathrm{B}}-\beta_{0} \mathbf{1}_{n_{\mathrm{B}}}-\boldsymbol{x}_{\mathrm{B}} \boldsymbol{\beta}\right)\right\} \\
& {\left[\psi \mid \boldsymbol{y}_{\mathrm{A}}, \boldsymbol{x}_{\mathrm{A}}, \boldsymbol{w}_{\mathrm{A}}, \boldsymbol{y}_{\mathrm{B}}, \boldsymbol{x}_{\mathrm{B}}, \boldsymbol{w}_{\mathrm{B}}, \boldsymbol{\beta}, \beta_{0}, \sigma^{2}, \nu, \tau^{2}, \boldsymbol{\mu}_{\boldsymbol{X}}, \boldsymbol{\Sigma}_{\boldsymbol{X}}^{-1}\right]} \\
& \propto\left[\boldsymbol{w}_{\mathrm{A}} \mid \boldsymbol{x}_{\mathrm{A}}, \psi, \nu, \tau^{2}\right]\left[\boldsymbol{w}_{\mathrm{B}} \mid \boldsymbol{x}_{\mathrm{B}}, \psi, \nu, \tau^{2}\right] \\
& \propto \exp \left\{-\frac{1}{2 \tau^{2}} \operatorname{Tr}\left(\boldsymbol{w}_{\mathrm{A}}-\psi \mathbf{1}_{n_{\mathrm{A}}} \mathbf{1}_{p}^{\top}-\nu \boldsymbol{x}_{\mathrm{A}}\right)^{\top}\left(\boldsymbol{w}_{\mathrm{A}}-\psi \mathbf{1}_{n_{\mathrm{A}}} \mathbf{1}_{p}^{\top}-\nu \boldsymbol{x}_{\mathrm{A}}\right)\right\} \\
& \times \exp \left\{-\frac{1}{2 \tau^{2}} \operatorname{Tr}\left(\boldsymbol{w}_{\mathrm{B}}-\psi \mathbf{1}_{n_{\mathrm{B}}} \mathbf{1}_{p}^{\top}-\nu \boldsymbol{x}_{\mathrm{B}}\right)^{\top}\left(\boldsymbol{w}_{\mathrm{B}}-\psi \mathbf{1}_{n_{\mathrm{B}}} \mathbf{1}_{p}^{\top}-\nu \boldsymbol{x}_{\mathrm{B}}\right)\right\} \\
& \propto \exp \left\{-\frac{1}{2 \tau^{2}}\left(\psi^{2} \operatorname{Tr}\left[\mathbf{1}_{p} \mathbf{1}_{n_{\mathrm{A}}}^{\top} \mathbf{1}_{n_{\mathrm{A}}} \mathbf{1}_{p}^{\top}+\mathbf{1}_{p} \mathbf{1}_{n_{\mathrm{B}}}^{\top} \mathbf{1}_{n_{\mathrm{B}}} \mathbf{1}_{p}^{\top}\right]-2 \psi \operatorname{Tr}\left[\mathbf{1}_{p} \mathbf{1}_{n_{\mathrm{A}}}^{\top}\left(\boldsymbol{w}_{\mathrm{A}}-\nu \boldsymbol{x}_{\mathrm{A}}\right)+\mathbf{1}_{p} \mathbf{1}_{n_{\mathrm{B}}}^{\top}\left(\boldsymbol{w}_{\mathrm{B}}-\nu \boldsymbol{x}_{\mathrm{B}}\right)\right]\right)\right\} \\
& =N\left\{\frac{\mathbf{1}_{n_{\mathrm{A}}}^{\top}\left(\boldsymbol{w}_{\mathrm{A}}-\nu \boldsymbol{x}_{\mathrm{A}}\right) \mathbf{1}_{p}+\mathbf{1}_{n_{\mathrm{B}}}^{\top}\left(\boldsymbol{w}_{\mathrm{B}}-\nu \boldsymbol{x}_{\mathrm{B}}\right) \mathbf{1}_{p}}{\left(n_{\mathrm{A}}+n_{\mathrm{B}}\right) p}, \frac{\tau^{2}}{\left(n_{\mathrm{A}}+n_{\mathrm{B}}\right) p}\right\} \\
& {\left[\nu \mid \boldsymbol{y}_{\mathrm{A}}, \boldsymbol{x}_{\mathrm{A}}, \boldsymbol{w}_{\mathrm{A}}, \boldsymbol{y}_{\mathrm{B}}, \boldsymbol{x}_{\mathrm{B}}, \boldsymbol{w}_{\mathrm{B}}, \boldsymbol{\beta}, \beta_{0}, \sigma^{2}, \psi, \tau^{2}, \boldsymbol{\mu}_{\boldsymbol{X}}, \boldsymbol{\Sigma}_{\boldsymbol{X}}^{-1}\right]} \\
& \propto\left[\boldsymbol{w}_{\mathrm{A}} \mid \boldsymbol{x}_{\mathrm{A}}, \psi, \nu, \tau^{2}\right]\left[\boldsymbol{w}_{\mathrm{B}} \mid \boldsymbol{x}_{\mathrm{B}}, \psi, \nu, \tau^{2}\right] \\
& \propto \exp \left\{-\frac{1}{2 \tau^{2}} \operatorname{Tr}\left(\boldsymbol{w}_{\mathrm{A}}-\psi \mathbf{1}_{n_{\mathrm{A}}} \mathbf{1}_{p}^{\top}-\nu \boldsymbol{x}_{\mathrm{A}}\right)^{\top}\left(\boldsymbol{w}_{\mathrm{A}}-\psi \mathbf{1}_{n_{\mathrm{A}}} \mathbf{1}_{p}^{\top}-\nu \boldsymbol{x}_{\mathrm{A}}\right)\right\} \\
& \times \exp \left\{-\frac{1}{2 \tau^{2}} \operatorname{Tr}\left(\boldsymbol{w}_{\mathrm{B}}-\psi \mathbf{1}_{n_{\mathrm{B}}} \mathbf{1}_{p}^{\top}-\nu \boldsymbol{x}_{\mathrm{B}}\right)^{\top}\left(\boldsymbol{w}_{\mathrm{B}}-\psi \mathbf{1}_{n_{\mathrm{B}}} \mathbf{1}_{p}^{\top}-\nu \boldsymbol{x}_{\mathrm{B}}\right)\right\} \\
& \propto \exp \left\{-\frac{1}{2 \tau^{2}}\left(\nu^{2} \operatorname{Tr}\left[\boldsymbol{x}_{\mathrm{A}}^{\top} \boldsymbol{x}_{\mathrm{A}}+\boldsymbol{x}_{\mathrm{B}}^{\top} \boldsymbol{x}_{\mathrm{B}}\right]-2 \nu \operatorname{Tr}\left[\boldsymbol{x}_{\mathrm{A}}^{\top}\left(\boldsymbol{w}_{\mathrm{A}}-\psi \mathbf{1}_{n_{\mathrm{A}}} \mathbf{1}_{p}^{\top}\right)+\boldsymbol{x}_{\mathrm{B}}^{\top}\left(\boldsymbol{w}_{\mathrm{B}}-\psi \mathbf{1}_{n_{\mathrm{B}}} \mathbf{1}_{p}^{\top}\right)\right]\right)\right\} \\
& =N\left\{\frac{\operatorname{Tr}\left[\boldsymbol{x}_{\mathrm{A}}^{\top}\left(\boldsymbol{w}_{\mathrm{A}}-\psi \mathbf{1}_{n_{\mathrm{A}}} \mathbf{1}_{p}^{\top}\right)+\boldsymbol{x}_{\mathrm{B}}^{\top}\left(\boldsymbol{w}_{\mathrm{B}}-\psi \mathbf{1}_{n_{\mathrm{B}}} \mathbf{1}_{p}^{\top}\right)\right]}{\operatorname{Tr}\left[\boldsymbol{x}_{\mathrm{A}}^{\top} \boldsymbol{x}_{\mathrm{A}}+\boldsymbol{x}_{\mathrm{B}}^{\top} \boldsymbol{x}_{\mathrm{B}}\right]}, \frac{\tau^{2}}{\operatorname{Tr}\left[\boldsymbol{x}_{\mathrm{A}}^{\top} \boldsymbol{x}_{\mathrm{A}}+\boldsymbol{x}_{\mathrm{B}}^{\top} \boldsymbol{x}_{\mathrm{B}}\right]}\right\} \\
& {\left[\tau^{2} \mid \boldsymbol{y}_{\mathrm{A}}, \boldsymbol{x}_{\mathrm{A}}, \boldsymbol{w}_{\mathrm{A}}, \boldsymbol{y}_{\mathrm{B}}, \boldsymbol{x}_{\mathrm{B}}, \boldsymbol{w}_{\mathrm{B}}, \boldsymbol{\beta}, \beta_{0}, \sigma^{2}, \psi, \nu, \boldsymbol{\mu}_{\boldsymbol{X}}, \boldsymbol{\Sigma}_{\boldsymbol{X}}^{-1}\right]} \\
& \propto\left[\boldsymbol{w}_{\mathrm{A}} \mid \boldsymbol{x}_{\mathrm{A}}, \psi, \nu, \tau^{2}\right]\left[\boldsymbol{w}_{\mathrm{B}} \mid \boldsymbol{x}_{\mathrm{B}}, \psi, \nu, \tau^{2}\right] \\
& \propto\left(\tau^{2}\right)^{-\left(n_{\mathrm{A}}+n_{\mathrm{B}}\right) p / 2} \exp \left\{-\frac{1}{2 \tau^{2}} \operatorname{Tr}\left(\boldsymbol{w}_{\mathrm{A}}-\psi \mathbf{1}_{n_{\mathrm{A}}} \mathbf{1}_{p}^{\top}-\nu \boldsymbol{x}_{\mathrm{A}}\right)^{\top}\left(\boldsymbol{w}_{\mathrm{A}}-\psi \mathbf{1}_{n_{\mathrm{A}}} \mathbf{1}_{p}^{\top}-\nu \boldsymbol{x}_{\mathrm{A}}\right)\right\} \\
& \times \exp \left\{-\frac{1}{2 \tau^{2}} \operatorname{Tr}\left(\boldsymbol{w}_{\mathrm{B}}-\psi \mathbf{1}_{n_{\mathrm{B}}} \mathbf{1}_{p}^{\top}-\nu \boldsymbol{x}_{\mathrm{B}}\right)^{\top}\left(\boldsymbol{w}_{\mathrm{B}}-\psi \mathbf{1}_{n_{\mathrm{B}}} \mathbf{1}_{p}^{\top}-\nu \boldsymbol{x}_{\mathrm{B}}\right)\right\}\left(\tau^{2}\right)^{-1} \\
& =I G\left\{\frac{1}{2}\left(n_{\mathrm{A}}+n_{\mathrm{B}}\right) p,\right. \\
& \frac{1}{2} \operatorname{Tr}\left(\boldsymbol{w}_{\mathrm{A}}-\psi \mathbf{1}_{n_{\mathrm{A}}} \mathbf{1}_{p}^{\top}-\nu \boldsymbol{x}_{\mathrm{A}}\right)^{\top}\left(\boldsymbol{w}_{\mathrm{A}}-\psi \mathbf{1}_{n_{\mathrm{A}}} \mathbf{1}_{p}^{\top}-\nu \boldsymbol{x}_{\mathrm{A}}\right) \\
& \left.+\frac{1}{2} \operatorname{Tr}\left(\boldsymbol{w}_{\mathrm{B}}-\psi \mathbf{1}_{n_{\mathrm{B}}} \mathbf{1}_{p}^{\top}-\nu \boldsymbol{x}_{\mathrm{B}}\right)^{\top}\left(\boldsymbol{w}_{\mathrm{B}}-\psi \mathbf{1}_{n_{\mathrm{B}}} \mathbf{1}_{p}^{\top}-\nu \boldsymbol{x}_{\mathrm{B}}\right)\right\}
\end{aligned}
$$




$$
\begin{aligned}
& {\left[\boldsymbol{\mu}_{\boldsymbol{X}} \mid \boldsymbol{y}_{\mathrm{A}}, \boldsymbol{x}_{\mathrm{A}}, \boldsymbol{w}_{\mathrm{A}}, \boldsymbol{y}_{\mathrm{B}}, \boldsymbol{x}_{\mathrm{B}}, \boldsymbol{w}_{\mathrm{B}}, \boldsymbol{\beta}, \beta_{0}, \sigma^{2}, \psi, \nu, \tau^{2}, \boldsymbol{\Sigma}_{\boldsymbol{X}}^{-1}\right]} \\
& \propto\left[\boldsymbol{x}_{\mathrm{A}} \mid \boldsymbol{\mu}_{\boldsymbol{X}}, \boldsymbol{\Sigma}_{\boldsymbol{X}}^{-1}\right]\left[\boldsymbol{x}_{\mathrm{B}} \mid \boldsymbol{\mu}_{\boldsymbol{X}}, \boldsymbol{\Sigma}_{\boldsymbol{X}}^{-1}\right] \\
& \propto \exp \left\{-\frac{1}{2} \operatorname{Tr}\left(\boldsymbol{x}_{\mathrm{A}}-\mathbf{1}_{n_{\mathrm{A}}} \boldsymbol{\mu}_{\boldsymbol{X}}^{\top}\right) \boldsymbol{\Sigma}_{\boldsymbol{X}}^{-1}\left(\boldsymbol{x}_{\mathrm{A}}-\mathbf{1}_{n_{\mathrm{A}}} \boldsymbol{\mu}_{\boldsymbol{X}}^{\top}\right)^{\top}-\frac{1}{2} \operatorname{Tr}\left(\boldsymbol{x}_{\mathrm{B}}-\mathbf{1}_{n_{\mathrm{B}}} \boldsymbol{\mu}_{\boldsymbol{X}}^{\top}\right) \boldsymbol{\Sigma}_{\boldsymbol{X}}^{-1}\left(\boldsymbol{x}_{\mathrm{B}}-\mathbf{1}_{n_{\mathrm{B}}} \boldsymbol{\mu}_{\boldsymbol{X}}^{\top}\right)^{\top}\right\} \\
& \propto \exp \left\{-\frac{1}{2}\left(\left[n_{\mathrm{A}}+n_{\mathrm{B}}\right] \boldsymbol{\mu}_{\boldsymbol{X}}^{\top} \boldsymbol{\Sigma}_{\boldsymbol{X}}^{-1} \boldsymbol{\mu}_{\boldsymbol{X}}-2 \boldsymbol{\mu}_{\boldsymbol{X}}^{\top} \boldsymbol{\Sigma}_{\boldsymbol{X}}^{-1}\left[\boldsymbol{x}_{\mathrm{A}}^{\top} \mathbf{1}_{n_{\mathrm{A}}}+\boldsymbol{x}_{\mathrm{B}}^{\top} \mathbf{1}_{n_{\mathrm{B}}}\right]\right)\right\} \\
& =N_{p}\left\{\frac{\boldsymbol{x}_{\mathrm{A}}^{\top} \mathbf{1}_{n_{\mathrm{A}}}+\boldsymbol{x}_{\mathrm{B}}^{\top} \mathbf{1}_{n_{\mathrm{B}}}}{n_{\mathrm{A}}+n_{\mathrm{B}}}, \frac{1}{n_{\mathrm{A}}+n_{\mathrm{B}}} \boldsymbol{\Sigma}_{\boldsymbol{X}}\right\} \\
& {\left[\boldsymbol{\Sigma}_{\boldsymbol{X}}^{-1} \mid \boldsymbol{y}_{\mathrm{A}}, \boldsymbol{x}_{\mathrm{A}}, \boldsymbol{w}_{\mathrm{A}}, \boldsymbol{y}_{\mathrm{B}}, \boldsymbol{x}_{\mathrm{B}}, \boldsymbol{w}_{\mathrm{B}}, \boldsymbol{\beta}, \beta_{0}, \sigma^{2}, \psi, \nu, \tau^{2}, \boldsymbol{\mu}_{\boldsymbol{X}}\right]} \\
& \propto\left[\boldsymbol{x}_{\mathrm{A}} \mid \boldsymbol{\mu}_{\boldsymbol{X}}, \boldsymbol{\Sigma}_{\boldsymbol{X}}^{-1}\right]\left[\boldsymbol{x}_{\mathrm{B}} \mid \boldsymbol{\mu}_{\boldsymbol{X}}, \boldsymbol{\Sigma}_{\boldsymbol{X}}^{-1}\right]\left[\boldsymbol{\Sigma}_{\boldsymbol{X}}^{-1}\right] \\
& =\left|\boldsymbol{\Sigma}_{\boldsymbol{X}}^{-1}\right|^{\left(n_{\mathrm{A}}+n_{\mathrm{B}}\right) / 2} \exp \left\{-\frac{1}{2} \operatorname{Tr} \boldsymbol{\Sigma}_{\boldsymbol{X}}^{-1}\left[\left(\boldsymbol{x}_{\mathrm{A}}-\mathbf{1}_{n_{\mathrm{A}}} \boldsymbol{\mu}_{\boldsymbol{X}}^{\top}\right)^{\top}\left(\boldsymbol{x}_{\mathrm{A}}-\mathbf{1}_{n_{\mathrm{A}}} \boldsymbol{\mu}_{\boldsymbol{X}}^{\top}\right)+\left(\boldsymbol{x}_{\mathrm{B}}-\mathbf{1}_{n_{\mathrm{B}}} \boldsymbol{\mu}_{\boldsymbol{X}}^{\top}\right)^{\top}\left(\boldsymbol{x}_{\mathrm{B}}-\mathbf{1}_{n_{\mathrm{B}}} \boldsymbol{\mu}_{\boldsymbol{X}}^{\top}\right)\right]\right\} \\
& \times\left|\boldsymbol{\Sigma}_{\boldsymbol{X}}^{-1}\right|^{(2 p-1) / 2} \exp \left\{-\frac{2 p-1}{2} \operatorname{Tr}\left(\operatorname{diag}\left(\hat{\operatorname{Var}}\left[\boldsymbol{x}_{\mathrm{A}}\right]\right) \boldsymbol{\Sigma}_{\boldsymbol{X}}^{-1}\right)\right\} \\
& =W\left\{3 p+n_{\mathrm{A}}+n_{\mathrm{B}},\right. \\
& \left((2 p-1) \operatorname{diag}\left(\hat{\operatorname{Var}}\left[\boldsymbol{x}_{\mathrm{A}}\right]\right)\right. \\
& \left.\left.+\left(\boldsymbol{x}_{\mathrm{A}}-\mathbf{1}_{n_{\mathrm{A}}} \boldsymbol{\mu}_{\boldsymbol{X}}^{\top}\right)^{\top}\left(\boldsymbol{x}_{\mathrm{A}}-\mathbf{1}_{n_{\mathrm{A}}} \boldsymbol{\mu}_{\boldsymbol{X}}^{\top}\right)+\left(\boldsymbol{x}_{\mathrm{B}}-\mathbf{1}_{n_{\mathrm{B}}} \boldsymbol{\mu}_{\boldsymbol{X}}^{\top}\right)^{\top}\left(\boldsymbol{x}_{\mathrm{B}}-\mathbf{1}_{n_{\mathrm{B}}} \boldsymbol{\mu}_{\boldsymbol{X}}^{\top}\right)\right)^{-1}\right\}
\end{aligned}
$$

The Inverse-Gamma distribution, $I G(a, b)$, is parametrized to have mean $\frac{b}{a-1}$ and the Wishart distribution, $W(d, S)$ is parametrized to have mean $d S$.

HIERBETAS, EBBETAS Replace the Jeffreys prior on $\boldsymbol{\beta}$ with

$$
\left[\boldsymbol{\beta} \mid \sigma^{2}, \lambda\right] \propto\left(\frac{\lambda}{\sigma^{2}}\right)^{p / 2} \exp \left\{-\frac{1}{2} \frac{\lambda}{\sigma^{2}} \boldsymbol{\beta}^{\top} \boldsymbol{\beta}\right\},
$$


The following posterior steps are modified:

$$
\begin{aligned}
& {\left[\boldsymbol{\beta} \mid \boldsymbol{y}_{\mathrm{A}}, \boldsymbol{x}_{\mathrm{A}}, \boldsymbol{w}_{\mathrm{A}}, \boldsymbol{y}_{\mathrm{B}}, \boldsymbol{x}_{\mathrm{B}}, \boldsymbol{w}_{\mathrm{B}}, \beta_{0}, \sigma^{2}, \nu, \tau^{2}, \boldsymbol{\mu}_{\boldsymbol{X}}, \boldsymbol{\Sigma}_{\boldsymbol{X}}^{-1}, \lambda\right] } \propto\left[\boldsymbol{y}_{\mathrm{A}} \mid \boldsymbol{x}_{\mathrm{A}}, \boldsymbol{\beta}, \beta_{0}, \sigma^{2}\right]\left[\boldsymbol{y}_{\mathrm{B}} \mid \boldsymbol{x}_{\mathrm{B}}, \boldsymbol{\beta}, \beta_{0}, \sigma^{2}\right]\left[\boldsymbol{\beta} \mid \sigma^{2}, \lambda\right] \\
& \propto \exp \left\{-\frac{1}{2 \sigma^{2}}\left(\boldsymbol{y}_{\mathrm{A}}-\boldsymbol{x}_{\mathrm{A}} \boldsymbol{\beta}\right)^{\top}\left(\boldsymbol{y}_{\mathrm{A}}-\boldsymbol{x}_{\mathrm{A}} \boldsymbol{\beta}\right)-\frac{1}{2 \sigma^{2}}\left(\boldsymbol{y}_{\mathrm{B}}-\boldsymbol{x}_{\mathrm{B}} \boldsymbol{\beta}\right)^{\top}\left(\boldsymbol{y}_{\mathrm{B}}-\boldsymbol{x}_{\mathrm{B}} \boldsymbol{\beta}\right)\right\} \exp \left\{-\frac{\lambda}{2 \sigma^{2}} \boldsymbol{\beta}^{\top} \boldsymbol{\beta}\right\} \\
& \propto \exp \left\{-\frac{1}{2 \sigma^{2}}\left(\boldsymbol{\beta}^{\top}\left[\boldsymbol{x}_{\mathrm{A}}^{\top} \boldsymbol{x}_{\mathrm{A}}+\boldsymbol{x}_{\mathrm{B}}^{\top} \boldsymbol{x}_{\mathrm{B}}+\lambda \boldsymbol{I}_{p}\right] \boldsymbol{\beta}-2 \boldsymbol{\beta}^{\top}\left[\boldsymbol{x}_{\mathrm{A}}^{\top}\left(\boldsymbol{y}_{\mathrm{A}}-\beta_{0} \mathbf{1}_{n_{\mathrm{A}}}\right)+\boldsymbol{x}_{\mathrm{B}}^{\top}\left(\boldsymbol{y}_{\mathrm{B}}-\beta_{0} \mathbf{1}_{n_{\mathrm{B}}}\right)\right]\right)\right\} \\
&= N_{p}\left\{\left(\boldsymbol{x}_{\mathrm{A}}^{\top} \boldsymbol{x}_{\mathrm{A}}+\boldsymbol{x}_{\mathrm{B}}^{\top} \boldsymbol{x}_{\mathrm{B}}+\lambda \boldsymbol{I}_{p}\right)^{-1}\left(\boldsymbol{x}_{\mathrm{A}}^{\top}\left[\boldsymbol{y}_{\mathrm{A}}-\beta_{0} \mathbf{1}_{n_{\mathrm{A}}}\right]+\boldsymbol{x}_{\mathrm{B}}^{\top}\left[\boldsymbol{y}_{\mathrm{B}}-\beta_{0} \mathbf{1}_{n_{\mathrm{B}}}\right]\right), \sigma^{2}\left(\boldsymbol{x}_{\mathrm{A}}^{\top} \boldsymbol{x}_{\mathrm{A}}+\boldsymbol{x}_{\mathrm{B}}^{\top} \boldsymbol{x}_{\mathrm{B}}+\lambda \boldsymbol{I}_{p}\right)^{-1}\right\} \\
& {\left[\sigma^{2} \mid \boldsymbol{y}_{\mathrm{A}}, \boldsymbol{x}_{\mathrm{A}}, \boldsymbol{w}_{\mathrm{A}}, \boldsymbol{y}_{\mathrm{B}}, \boldsymbol{x}_{\mathrm{B}}, \boldsymbol{w}_{\mathrm{B}}, \boldsymbol{\beta}, \beta_{0}, \nu, \tau^{2}, \boldsymbol{\mu}_{\boldsymbol{X}}, \boldsymbol{\Sigma}_{\boldsymbol{X}}^{-1}, \lambda\right] } \\
& \propto\left[\boldsymbol{y}_{\mathrm{A}} \mid \boldsymbol{x}_{\mathrm{A}}, \boldsymbol{\beta}, \beta_{0}, \sigma^{2}\right]\left[\boldsymbol{y}_{\mathrm{B}} \mid \boldsymbol{x}_{\mathrm{B}}, \boldsymbol{\beta}, \beta_{0}, \sigma^{2}\right]\left[\boldsymbol{\beta} \mid \sigma^{2}, \lambda\right]\left[\sigma^{2}\right] \\
& \propto\left(\sigma^{2}\right)^{-n_{\mathrm{A}} / 2} \exp \left\{-\frac{1}{2 \sigma^{2}}\left(\boldsymbol{y}_{\mathrm{A}}-\beta_{0} \mathbf{1}_{n_{\mathrm{A}}}-\boldsymbol{x}_{\mathrm{A}} \boldsymbol{\beta}\right)^{\top}\left(\boldsymbol{y}_{\mathrm{A}}-\beta_{0} \mathbf{1}_{n_{\mathrm{A}}}-\boldsymbol{x}_{\mathrm{A}} \boldsymbol{\beta}\right)\right\} \\
& \quad \times\left(\sigma^{2}\right)^{-n_{\mathrm{B}} / 2} \exp \left\{-\frac{1}{2 \sigma^{2}}\left(\boldsymbol{y}_{\mathrm{B}}-\beta_{0} \mathbf{1}_{n_{\mathrm{B}}}-\boldsymbol{x}_{\mathrm{B}} \boldsymbol{\beta}\right)^{\top}\left(\boldsymbol{y}_{\mathrm{B}}-\beta_{0} \mathbf{1}_{n_{\mathrm{B}}}-\boldsymbol{x}_{\mathrm{B}} \boldsymbol{\beta}\right)\right\}\left(\sigma^{2}\right)^{-1} \\
& \quad \times\left(\sigma^{2}\right)^{-p / 2} \exp \left\{-\frac{\lambda}{2 \sigma^{2}} \boldsymbol{\beta}^{\top} \boldsymbol{\beta}\right\} \\
&=I G\left\{\frac{1}{2}\left(n_{\mathrm{A}}+n_{\mathrm{B}}+p\right),\right. \\
& \quad \frac{1}{2} \lambda \boldsymbol{\beta}^{\top} \boldsymbol{\beta}+\frac{1}{2}\left(\boldsymbol{y}_{\mathrm{A}}-\beta_{0} \mathbf{1}_{n_{\mathrm{A}}}-\boldsymbol{x}_{\mathrm{A}} \boldsymbol{\beta}\right)^{\top}\left(\boldsymbol{y}_{\mathrm{A}}-\beta_{0} \mathbf{1}_{n_{\mathrm{A}}}-\boldsymbol{x}_{\mathrm{A}} \boldsymbol{\beta}\right) \\
&\left.\quad+\frac{1}{2}\left(\boldsymbol{y}_{\mathrm{B}}-\beta_{0} \mathbf{1}_{n_{\mathrm{B}}}-\boldsymbol{x}_{\mathrm{B}} \boldsymbol{\beta}\right)^{\top}\left(\boldsymbol{y}_{\mathrm{B}}-\beta_{0} \mathbf{1}_{n_{\mathrm{B}}}-\boldsymbol{x}_{\mathrm{B}} \boldsymbol{\beta}\right)\right\}
\end{aligned}
$$

The HIERBETAS update for $\lambda$ is given as follows:

$$
\begin{aligned}
& {\left[\lambda \mid \boldsymbol{y}_{\mathrm{A}}, \boldsymbol{x}_{\mathrm{A}}, \boldsymbol{w}_{\mathrm{A}}, \boldsymbol{y}_{\mathrm{B}}, \boldsymbol{x}_{\mathrm{B}}, \boldsymbol{w}_{\mathrm{B}}, \boldsymbol{\beta}, \beta_{0}, \sigma^{2}, \nu, \tau^{2}, \boldsymbol{\mu}_{\boldsymbol{X}}, \boldsymbol{\Sigma}_{\boldsymbol{X}}^{-1}\right]} \\
& \quad \propto\left[\boldsymbol{\beta} \mid \sigma^{2}, \lambda\right][\lambda] \\
& \quad \propto \lambda^{p / 2} \exp \left\{-\frac{\lambda}{2 \sigma^{2}} \boldsymbol{\beta}^{\top} \boldsymbol{\beta}\right\} \lambda^{-1} \\
& \quad=\mathcal{G}\left\{\frac{p}{2}, \frac{\boldsymbol{\beta}^{\top} \boldsymbol{\beta}}{2 \sigma^{2}}\right\}
\end{aligned}
$$

To calculate the EBBETAS update for $\lambda$, observe that $\mathrm{E}_{\boldsymbol{\phi} \mid \boldsymbol{U}^{\mathrm{obs}}, \lambda} \ln \left[\boldsymbol{\beta} \mid \sigma^{2}, \lambda\right]=(p / 2) \ln \lambda-\lambda \mathrm{E}\left[\boldsymbol{\beta}^{\top} \boldsymbol{\beta} / \sigma^{2}\right] / 2$. This is maximized with respect to $\lambda$ when $\lambda=p / \mathrm{E}\left[\boldsymbol{\beta}^{\top} \boldsymbol{\beta} / \sigma^{2}\right]$.

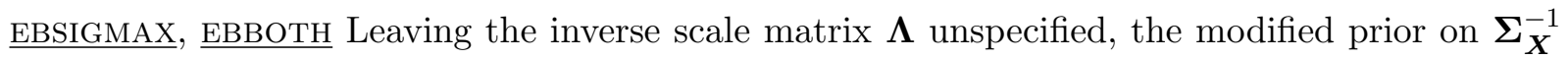
is

$$
\left[\boldsymbol{\Sigma}_{\boldsymbol{X}}^{-1} \mid \boldsymbol{\Lambda}\right] \propto|\boldsymbol{\Lambda}|^{3 p / 2}\left|\boldsymbol{\Sigma}_{\boldsymbol{X}}^{-1}\right|^{(2 p-1) / 2} \exp \left\{-(1 / 2) \operatorname{Tr}\left(\boldsymbol{\Lambda} \boldsymbol{\Sigma}_{\boldsymbol{X}}^{-1}\right)\right\}
$$


$\boldsymbol{\Lambda}$ is the (unknown) positive-definite matrix of hyperparameters. The Gibbs step for $\boldsymbol{\Sigma}_{\boldsymbol{X}}^{-1}$ becomes

$$
\begin{aligned}
& {\left[\boldsymbol{\Sigma}_{\boldsymbol{X}}^{-1} \mid \boldsymbol{y}_{\mathrm{A}}, \boldsymbol{x}_{\mathrm{A}}, \boldsymbol{w}_{\mathrm{A}}, \boldsymbol{y}_{\mathrm{B}}, \boldsymbol{x}_{\mathrm{B}}, \boldsymbol{w}_{\mathrm{B}}, \boldsymbol{\beta}, \beta_{0}, \sigma^{2}, \psi, \nu, \tau^{2}, \boldsymbol{\mu}_{\boldsymbol{X}}\right]} \\
& \propto\left[\boldsymbol{x}_{\mathrm{A}} \mid \boldsymbol{\mu}_{\boldsymbol{X}}, \boldsymbol{\Sigma}_{\boldsymbol{X}}^{-1}\right]\left[\boldsymbol{x}_{\mathrm{B}} \mid \boldsymbol{\mu}_{\boldsymbol{X}}, \boldsymbol{\Sigma}_{\boldsymbol{X}}^{-1}\right]\left[\boldsymbol{\Sigma}_{\boldsymbol{X}}^{-1} \mid \boldsymbol{\Lambda}\right] \\
& \propto\left|\boldsymbol{\Sigma}_{\boldsymbol{X}}^{-1}\right|^{\left(n_{\mathrm{A}}+n_{\mathrm{B}}\right) / 2}\left\{-\frac{1}{2} \operatorname{Tr}\left(\boldsymbol{x}_{\mathrm{A}}-\mathbf{1}_{n_{\mathrm{A}}} \boldsymbol{\mu}_{\boldsymbol{X}}^{\top}\right) \boldsymbol{\Sigma}_{\boldsymbol{X}}^{-1}\left(\boldsymbol{x}_{\mathrm{A}}-\mathbf{1}_{n_{\mathrm{A}}} \boldsymbol{\mu}_{\boldsymbol{X}}^{\top}\right)^{\top}\right\} \\
& \quad \times \exp \left\{-\frac{1}{2} \operatorname{Tr}\left(\boldsymbol{x}_{\mathrm{B}}-\mathbf{1}_{n_{\mathrm{B}}} \boldsymbol{\mu}_{\boldsymbol{X}}^{\top}\right) \boldsymbol{\Sigma}_{\boldsymbol{X}}^{-1}\left(\boldsymbol{x}_{\mathrm{B}}-\mathbf{1}_{n_{\mathrm{B}}} \boldsymbol{\mu}_{\boldsymbol{X}}^{\top}\right)^{\top}\right\} \\
& \quad \times\left|\boldsymbol{\Sigma}_{\boldsymbol{X}}^{-1}\right|^{(2 p-1) / 2} \exp \left\{-\frac{1}{2} \operatorname{Tr}\left(\boldsymbol{\Lambda} \boldsymbol{\Sigma}_{\boldsymbol{X}}^{-1}\right)\right\} \\
& =W\left\{3 p+n_{\mathrm{A}}+n_{\mathrm{B}},\right. \\
& \left.\quad \quad\left(\boldsymbol{\Lambda}+\left(\boldsymbol{x}_{\mathrm{A}}-\mathbf{1}_{n_{\mathrm{A}}} \boldsymbol{\mu}_{\boldsymbol{X}}^{\top}\right)^{\top}\left(\boldsymbol{x}_{\mathrm{A}}-\mathbf{1}_{n_{\mathrm{A}}} \boldsymbol{\mu}_{\boldsymbol{X}}^{\top}\right)+\left(\boldsymbol{x}_{\mathrm{B}}-\mathbf{1}_{n_{\mathrm{B}}} \boldsymbol{\mu}_{\boldsymbol{X}}^{\top}\right)^{\top}\left(\boldsymbol{x}_{\mathrm{B}}-\mathbf{1}_{n_{\mathrm{B}}} \boldsymbol{\mu}_{\boldsymbol{X}}^{\top}\right)\right)^{-1}\right\}
\end{aligned}
$$

We now derive the Empirical Bayes update for the diagonal inverse-scale matrix $\boldsymbol{\Lambda}=\operatorname{diag}\left\{\Lambda_{11}, \ldots, \Lambda_{p p}\right\}$. Observe that

$$
\mathrm{E}_{\boldsymbol{\phi} \mid \boldsymbol{U}^{\mathrm{obs}, \boldsymbol{\Lambda}}} \ln \left[\boldsymbol{\Sigma}_{\boldsymbol{X}}^{-1} \mid \boldsymbol{\Lambda}\right] \propto p \ln |\boldsymbol{\Lambda}|-\operatorname{Tr}\left(\boldsymbol{\Lambda} \mathrm{E}\left[\boldsymbol{\Sigma}_{\boldsymbol{X}}^{-1}\right]\right)=3 p \sum_{i=1}^{p} \log \Lambda_{i i}-\sum_{i=1}^{p} \Lambda_{i i} \mathrm{E}\left[\boldsymbol{\Sigma}_{\boldsymbol{X}}^{-1}\right]_{i i}
$$

Thus, each element $\Lambda_{i i}$ may be optimized individually, yielding the Empirical Bayes update $\Lambda_{i i} \leftarrow$ $3 p \mathrm{E}\left[\boldsymbol{\Sigma}_{\boldsymbol{X}}^{-1}\right]_{i i}^{-1}$

APPENDIX B: MODIFIED GIBBS STEPS FOR DATA ANALYSIS

Let $\boldsymbol{\psi} \equiv\left\{\psi_{1}, \ldots, \psi_{p}\right\}, \boldsymbol{\nu} \equiv \operatorname{diag}\left\{\nu_{1}, \ldots, \nu_{p}\right\}$ (that is, a diagonal matrix with components $\left.\nu_{1}, \ldots, \nu_{p}\right)$, and $\left\{\boldsymbol{e}_{j}\right\}$ the set of $p$-dimensional standard basic vectors. The conditional distributions 
with individual intercepts and slopes (using flat priors on each $\psi_{j}$ and $\nu_{j}$ ) are given by

$$
\begin{aligned}
& {[\boldsymbol{\psi} \mid}\left.\boldsymbol{y}_{\mathrm{A}}, \boldsymbol{x}_{\mathrm{A}}, \boldsymbol{w}_{\mathrm{A}}, \boldsymbol{y}_{\mathrm{B}}, \boldsymbol{x}_{\mathrm{B}}, \boldsymbol{w}_{\mathrm{B}}, \boldsymbol{\beta}, \beta_{0}, \sigma^{2}, \boldsymbol{\nu}, \tau^{2}, \boldsymbol{\mu}_{\boldsymbol{X}}, \boldsymbol{\Sigma}_{\boldsymbol{X}}^{-1}\right] \\
& \propto {\left[\boldsymbol{w}_{\mathrm{A}} \mid \boldsymbol{x}_{\mathrm{A}}, \boldsymbol{\psi}, \boldsymbol{\nu}, \tau^{2}\right]\left[\boldsymbol{w}_{\mathrm{B}} \mid \boldsymbol{x}_{\mathrm{B}}, \boldsymbol{\psi}, \boldsymbol{\nu}, \tau^{2}\right] } \\
& \propto \exp \left\{-\frac{1}{2 \tau^{2}} \operatorname{Tr}\left(\boldsymbol{w}_{\mathrm{A}}-\mathbf{1}_{n_{\mathrm{A}}} \boldsymbol{\psi}^{\top}-\boldsymbol{x}_{\mathrm{A}} \boldsymbol{\nu}\right)^{\top}\left(\boldsymbol{w}_{\mathrm{A}}-\mathbf{1}_{n_{\mathrm{A}}} \boldsymbol{\psi}^{\top}-\boldsymbol{x}_{\mathrm{A}} \boldsymbol{\nu}\right)\right\} \\
& \times \exp \left\{-\frac{1}{2 \tau^{2}} \operatorname{Tr}\left(\boldsymbol{w}_{\mathrm{B}}-\mathbf{1}_{n_{\mathrm{B}}} \boldsymbol{\psi}^{\top}-\boldsymbol{x}_{\mathrm{B}} \boldsymbol{\nu}\right)^{\top}\left(\boldsymbol{w}_{\mathrm{B}}-\mathbf{1}_{n_{\mathrm{B}}} \boldsymbol{\psi}^{\top}-\boldsymbol{x}_{\mathrm{B}} \boldsymbol{\nu}\right)\right\} \\
& \propto \exp \left\{-\frac{1}{2 \tau^{2}}\left(\operatorname{Tr}\left[\boldsymbol{\psi} \mathbf{1}_{n_{\mathrm{A}}}^{\top} \mathbf{1}_{n_{\mathrm{A}}} \boldsymbol{\psi}^{\top}+\boldsymbol{\psi} \mathbf{1}_{n_{\mathrm{B}}}^{\top} \mathbf{1}_{n_{\mathrm{B}}} \boldsymbol{\psi}^{\top}\right]-2 \operatorname{Tr}\left[\boldsymbol{\psi} \mathbf{1}_{n_{\mathrm{A}}}^{\top}\left(\boldsymbol{w}_{\mathrm{A}}-\boldsymbol{x}_{\mathrm{A}} \boldsymbol{\nu}\right)+\boldsymbol{\psi} \mathbf{1}_{n_{\mathrm{B}}}^{\top}\left(\boldsymbol{w}_{\mathrm{B}}-\boldsymbol{x}_{\mathrm{B}} \boldsymbol{\nu}\right)\right]\right)\right\} \\
&= \exp \left\{-\frac{1}{2 \tau^{2}}\left(\left(n_{\mathrm{A}}+n_{\mathrm{B}}\right) \sum_{j=1}^{p} \psi_{j}^{2}-\sum_{j=1}^{p} \psi_{j} \boldsymbol{e}_{j}^{\top}\left[\left(\boldsymbol{w}_{\mathrm{A}}-\boldsymbol{x}_{\mathrm{A}} \boldsymbol{\nu}\right)^{\top} \mathbf{1}_{n_{\mathrm{A}}}+\left(\boldsymbol{w}_{\mathrm{B}}-\boldsymbol{x}_{\mathrm{B}} \boldsymbol{\nu}\right)^{\top} \mathbf{1}_{n_{\mathrm{B}}}\right]\right)\right\} \\
& {\left[\nu \mid \boldsymbol{y}_{\mathrm{A}}, \boldsymbol{x}_{\mathrm{A}}, \boldsymbol{w}_{\mathrm{A}}, \boldsymbol{y}_{\mathrm{B}}, \boldsymbol{x}_{\mathrm{B}}, \boldsymbol{w}_{\mathrm{B}}, \boldsymbol{\beta}, \beta_{0}, \sigma^{2}, \psi, \tau^{2}, \boldsymbol{\mu}_{\boldsymbol{X}}, \boldsymbol{\Sigma}_{\boldsymbol{X}}^{-1}\right] } \\
& \propto\left[\boldsymbol{w}_{\mathrm{A}} \mid \boldsymbol{x}_{\mathrm{A}}, \psi, \nu, \tau^{2}\right]\left[\boldsymbol{w}_{\mathrm{B}} \mid \boldsymbol{x}_{\mathrm{B}}, \psi, \nu, \tau^{2}\right] \\
& \propto \exp \left\{-\frac{1}{2 \tau^{2}} \operatorname{Tr}\left(\boldsymbol{w}_{\mathrm{A}}-\mathbf{1}_{n_{\mathrm{A}}} \boldsymbol{\psi}^{\top}-\boldsymbol{x}_{\mathrm{A}} \boldsymbol{\nu}\right)^{\top}\left(\boldsymbol{w}_{\mathrm{A}}-\mathbf{1}_{n_{\mathrm{A}}} \boldsymbol{\psi}^{\top}-\boldsymbol{x}_{\mathrm{A}} \boldsymbol{\nu}\right)\right\} \\
& \quad \times \exp \left\{-\frac{1}{2 \tau^{2}} \operatorname{Tr}\left(\boldsymbol{w}_{\mathrm{B}}-\mathbf{1}_{n_{\mathrm{B}}} \boldsymbol{\psi}^{\top}-\boldsymbol{x}_{\mathrm{B}} \boldsymbol{\nu}\right)^{\top}\left(\boldsymbol{w}_{\mathrm{B}}-\mathbf{1}_{n_{\mathrm{B}}} \boldsymbol{\psi}^{\top}-\boldsymbol{x}_{\mathrm{B}} \boldsymbol{\nu}\right)\right\} \\
& \quad \propto \exp \left\{-\frac{1}{2 \tau^{2}}\left(\operatorname{Tr}\left[\boldsymbol{\nu}^{2} \boldsymbol{x}_{\mathrm{A}}^{\top} \boldsymbol{x}_{\mathrm{A}}+\boldsymbol{\nu}^{2} \boldsymbol{x}_{\mathrm{B}}^{\top} \boldsymbol{x}_{\mathrm{B}}\right]-2 \operatorname{Tr}\left[\boldsymbol{\nu} \boldsymbol{x}_{\mathrm{A}}^{\top}\left(\boldsymbol{w}_{\mathrm{A}}-\mathbf{1}_{n_{\mathrm{A}}} \boldsymbol{\psi}^{\top}\right)+\boldsymbol{\nu} \boldsymbol{x}_{\mathrm{B}}^{\top}\left(\boldsymbol{w}_{\mathrm{B}}-\mathbf{1}_{n_{\mathrm{B}}} \boldsymbol{\psi}^{\top}\right)\right]\right)\right\}
\end{aligned}
$$

From these, the modified Gibbs steps are

$$
\begin{aligned}
& \psi_{j} \leftarrow N\left\{\frac{\boldsymbol{e}_{j}^{\top}\left[\left(\boldsymbol{w}_{\mathrm{A}}-\boldsymbol{x}_{\mathrm{A}} \boldsymbol{\nu}\right)^{\top} \mathbf{1}_{n_{\mathrm{A}}}+\left(\boldsymbol{w}_{\mathrm{B}}-\boldsymbol{x}_{\mathrm{B}} \boldsymbol{\nu}\right)^{\top} \mathbf{1}_{n_{\mathrm{B}}}\right]}{\left(n_{\mathrm{A}}+n_{\mathrm{B}}\right)}, \frac{\tau^{2}}{\left(n_{\mathrm{A}}+n_{\mathrm{B}}\right)}\right\} \\
& \nu_{j} \leftarrow N\left\{\frac{\boldsymbol{e}_{j}^{\top}\left[\boldsymbol{x}_{\mathrm{A}}^{\top}\left(\boldsymbol{w}_{\mathrm{A}}-\mathbf{1}_{n_{\mathrm{A}}} \boldsymbol{\psi}^{\top}\right)+\boldsymbol{x}_{\mathrm{B}}^{\top}\left(\boldsymbol{w}_{\mathrm{B}}-\mathbf{1}_{n_{\mathrm{B}}} \boldsymbol{\psi}^{\top}\right)\right] \boldsymbol{e}_{j}}{\boldsymbol{e}_{j}^{\top}\left[\boldsymbol{x}_{\mathrm{A}}^{\top} \boldsymbol{x}_{\mathrm{A}}+\boldsymbol{x}_{\mathrm{B}}^{\top} \boldsymbol{x}_{\mathrm{B}}\right] \boldsymbol{e}_{j}}, \frac{\tau^{2}}{\boldsymbol{e}_{j}^{\top}\left[\boldsymbol{x}_{\mathrm{A}}^{\top} \boldsymbol{x}_{\mathrm{A}}+\boldsymbol{x}_{\mathrm{B}}^{\top} \boldsymbol{x}_{\mathrm{B}}\right] \boldsymbol{e}_{j}}\right\}
\end{aligned}
$$

independently for $j=1, \ldots, p$.

\section{APPENDIX C: DESCRIPTION OF PREPROCESSING STEPS FOR DATA ANALYSIS}

The data were first normalized according to the prescription of Chen et al. (2011). 11 measurements in the qRT-PCR-only data, out of $47 \times 91=4277$ total, or 0.26 percent, were missing; in order to use all observations, these values were imputed using chained equations (Su et al., 2011) and thereafter assumed known. Bayesian methods of imputation, like those discussed for imputing $\boldsymbol{x}_{\mathrm{B}}$, are a better approach to handle this missingness but, given the small percentage of missingness, would likely not affect the results. Additionally, four tumors, three in the Affymetrix-only sample and one in the validation sample, had event times less than one month after surgery; these were removed before analysis. Thus $n_{\mathrm{A}}=47, n_{\mathrm{B}}=389$, and the validation sample is size 100 . 


\section{APPENDIX D: COMPUTATIONAL DETAILS}

The Gibbs sampler code was written in the $\mathrm{C}$ language and called using $\mathrm{R}$ ( $\mathrm{R}$ Core Team, 2012). The $R$ environment was also used for all analyses. The simulation study in Section 4 of the manuscript was run on a cluster of 29 nodes with 392 cores, comprised of Intel Xeon X5660 and other Intel-based processors, each with $32 \mathrm{~GB}$ of memory. With this configuration, using a burn-in period of 2500 iterations and 3500 total iterations, it took approximately 110 seconds to fit each Bayesian method to each simulated dataset. The data analysis in Section 5 of the manuscript was run on an Intel Core i7-3520M processor with 8GB of memory. With a burnin-period of 4000 iterations and 8000 total iterations, it took between 268-269 seconds to fit each Bayesian method.

Figures 2 and 3 in the manuscript were created with the R package lattice (Sarkar, 2008). The Scaled Integrated Brier Scores in Table 3 of the manuscript were calculated using code based on the $\mathrm{R}$ package ipred (Peters and Hothorn, 2012).

\section{REFERENCES}

Chen, G., Kim, S., Taylor, J. M. G., Wang, Z., Lee, O., Ramnath, N., Reddy, R. M., Lin, J., Chang, A. C., OrRinger, M. B. and BeER, D. G. (2011). Development and validation of a qRT-PCR-classifier for lung cancer prognosis. Journal of Thoracic Oncology 6 1481-1487.

Peters, A. and Hothorn, T. (2012). ipred: Improved Predictors R package version 0.9-1.

R Core Team, (2012). R: A Language and Environment for Statistical Computing, Vienna, Austria.

SARKAR, D. (2008). Lattice: Multivariate Data Visualization with R. Springer, New York.

Su, Y.-S., Gelman, A., Hill, J. and Yajima, M. (2011). Multiple Imputation with Diagnostics (mi) in R: Opening Windows into the Black Box. Journal of Statistical Software 45 1-31. 


\begin{tabular}{|c|c|c|c|c|c|c|c|c|c|c|c|c|}
\hline \multirow[b]{2}{*}{$\rho, R^{2}$} & \multirow[b]{2}{*}{$n_{\mathrm{B}}$} & \multirow[b]{2}{*}{ Method } & \multicolumn{5}{|c|}{$\operatorname{MSPE}\left(\hat{\boldsymbol{\beta}}^{\mathrm{ppm}}\right)$} & \multicolumn{5}{|c|}{$\operatorname{MSPE}\left(\hat{\boldsymbol{\beta}}^{\mathrm{pm}}\right)$} \\
\hline & & & $\tau=0.01$ & 0.5 & $\quad 1.0$ & 1.50 & 2.00 & 0.01 & 0.5 & 1.0 & 1.50 & 2.00 \\
\hline \multirow[t]{6}{*}{$0.15,0.1$} & 400 & RIDG & 700.2 & 691.4 & 694.4 & 697.8 & 692.7 & 700.2 & 691.4 & 694.4 & 697.8 & 692.7 \\
\hline & & VANILLA & 793.2 & 888.6 & 1074.8 & 1387.1 & 1499.2 & 793.2 & 919.6 & 1215.8 & 1566.4 & 1656.3 \\
\hline & & HIERBETAS & 637.1 & 640.8 & 641.4 & 643.4 & 647.3 & 637.1 & 640.5 & 640.2 & 641.7 & 645.1 \\
\hline & & EBBETAS & 643.8 & 645.7 & 645.4 & 648.1 & 648.4 & 643.8 & 645.4 & 644.4 & 646.7 & 646.8 \\
\hline & & EBSIGMAX & 793.2 & 878.5 & 1092.5 & 1432.2 & 1573.0 & 793.1 & 906.8 & 1238.2 & 1625.4 & 1744.6 \\
\hline & & ЕВВОТН & 644.1 & 645.8 & 645.3 & 648.1 & 648.8 & 644.1 & 645.5 & 644.3 & 646.8 & 647.1 \\
\hline \multirow[t]{6}{*}{$0.75,0.1$} & 400 & RIDG & 312.2 & 323.4 & 311.5 & 311.7 & 314.4 & 312.2 & 323.4 & 311.5 & 311.7 & 314.4 \\
\hline & & VANILLA & 347.6 & 341.9 & 361.3 & 381.8 & 399.9 & 347.6 & 348.8 & 386.2 & 429.6 & 462.7 \\
\hline & & HIERBETAS & 281.3 & 282.6 & 283.3 & 284.6 & 287.3 & 281.3 & 282.6 & 283.2 & 284.4 & 287.1 \\
\hline & & EBBETAS & 286.6 & 288.4 & 287.8 & 289.0 & 291.6 & 286.6 & 288.4 & 287.7 & 288.8 & 291.4 \\
\hline & & EBSIGMAX & 347.7 & 369.0 & 396.6 & 437.4 & 478.2 & 347.7 & 381.9 & 435.8 & 504.8 & 563.0 \\
\hline & & ЕВВотн & 286.7 & 288.5 & 288.1 & 289.9 & 292.3 & 286.7 & 288.5 & 288.0 & 289.6 & 292.0 \\
\hline \multirow[t]{6}{*}{$0.15,0.4$} & 400 & RIDG & 124.7 & 125.2 & 125.2 & 125.7 & 127.4 & 124.7 & 125.2 & 125.2 & 125.7 & 127.4 \\
\hline & & VANILLA & 132.5 & 149.0 & 186.9 & 235.2 & 265.5 & 132.5 & 154.4 & 211.3 & 266.2 & 293.1 \\
\hline & & HIERBETAS & 111.2 & 112.3 & 113.9 & 115.2 & 117.4 & 111.2 & 112.2 & 113.7 & 114.9 & 117.0 \\
\hline & & EBBETAS & 111.1 & 112.0 & 113.5 & 114.5 & 116.1 & 111 & 112.0 & 113.3 & 114.1 & 115.6 \\
\hline & & EBSIGMAX & 132.5 & 147.3 & 188.9 & 246.7 & 283.5 & 132.5 & 152.2 & 214.6 & 278.8 & 311.5 \\
\hline & & ЕВВОтН & 111.1 & 112.0 & 113.5 & 114.5 & 116.3 & 111.1 & 111.9 & 113.3 & 114.1 & 115.8 \\
\hline \multirow[t]{6}{*}{$0.75,0.4$} & 400 & RIDG & 62.3 & 62.6 & 62.0 & 61.8 & 62.5 & 62.3 & 62.6 & 62.0 & 61.8 & 62.5 \\
\hline & & VANILLA & 58.1 & 57.6 & 61.2 & 68.0 & 73.1 & 58.1 & 58.7 & 65.4 & 77.2 & 85.3 \\
\hline & & HIERBETAS & 49.1 & 49.7 & 50.2 & 51.3 & 53.2 & 49.1 & 49.7 & 50.2 & 51.4 & 53.5 \\
\hline & & EBBETAS & 49.2 & 49.8 & 50.4 & 51.2 & 53.1 & 49.2 & 49.8 & 50.4 & 51.3 & 53.3 \\
\hline & & EBSIGMAX & 58.1 & 62.1 & 67.1 & 79.3 & 88.8 & 58.1 & 64.3 & 73.9 & 92.4 & 104.1 \\
\hline & & ЕВВотН & 49.2 & 49.8 & 50.7 & 51.8 & 54.0 & 49.2 & 49.8 & 50.7 & 51.9 & 54.1 \\
\hline \multirow[t]{6}{*}{$0.15,0.1$} & 150 & RIDG & 694.4 & 712.1 & 691.8 & 699.3 & 701.2 & 694.4 & 712.1 & 691.8 & 699.3 & 701.2 \\
\hline & & VANILLA & 1245.3 & 1505.9 & 2321.9 & 2256.3 & 2222.5 & 1245.2 & 1612.4 & 2492.4 & 2412.2 & 2351.4 \\
\hline & & HIERBETAS & 646.7 & 649.3 & 652.1 & 657.3 & 669.3 & 646.6 & 648.8 & 650.8 & 655.7 & 667.5 \\
\hline & & EBBETAS & 650.0 & 654.9 & 654.9 & 658.2 & 662.6 & 650.0 & 654.5 & 653.9 & 656.8 & 660.8 \\
\hline & & EBSIGMAX & 1245.5 & 1483.5 & 2478.8 & 2369.8 & 2236.5 & 1245.4 & 1585.2 & 2636.2 & 2514.1 & 2374.6 \\
\hline & & ЕВВОтн & 650.4 & 654.5 & 654.8 & 658.6 & 662.6 & 650.4 & 654.1 & 653.8 & 657.1 & 660.8 \\
\hline \multirow[t]{6}{*}{$0.75,0.1$} & 150 & RIDG & 322.3 & 317.6 & 316.2 & 311.9 & 320.4 & 322.3 & 317.6 & 316.2 & 311.9 & 320.4 \\
\hline & & VANILLA & 539.6 & 550.4 & 656.1 & 689.5 & 713.6 & 539.5 & 584.4 & 725.3 & 767.2 & 789.4 \\
\hline & & HIERBETAS & 287.2 & 289.0 & 289.5 & 291.8 & 294.8 & 287.2 & 288.9 & 289.2 & 291.6 & 294.9 \\
\hline & & EBBETAS & 291.9 & 292.9 & 292.1 & 294.9 & 296.7 & 291.9 & 292.8 & 291.9 & 294.7 & 296.7 \\
\hline & & EBSIGMAX & 539.3 & 644.0 & 793.7 & 5.8 & 858.9 & 39.3 & 697.5 & 868.2 & 896.8 & 941.5 \\
\hline & & ЕВВОТн & 92.0 & 293.1 & 292.6 & 295.4 & 297.4 & 292.0 & 293.0 & 292.4 & 295.2 & 297.3 \\
\hline \multirow[t]{6}{*}{$0.15,0.4$} & 150 & RIDG & 127.4 & 127.8 & 128.4 & 127.3 & 126.8 & 127.4 & 127.8 & 128.4 & 127.3 & 126.8 \\
\hline & & VANILLA & 207.4 & 250.5 & 404.2 & 395.8 & 394.9 & 207.4 & 267.8 & 429.6 & 419.7 & 418.7 \\
\hline & & HIERBETAS & 115.6 & 116.2 & 118.1 & 120.3 & 124.3 & 115.6 & 116.1 & 117.8 & 120.0 & 124.0 \\
\hline & & EBBETAS & 115.2 & 115.7 & 116.9 & 118.2 & 120.0 & 115.2 & 115.5 & 116.6 & 117.8 & 119.5 \\
\hline & & EBSIGMAX & 207.5 & 247.2 & 416.7 & & & & 264.3 & 443.2 & 9.6 & 431.3 \\
\hline & & ЕВВОтн & 115.2 & 115.6 & 116.9 & 118.3 & 120.2 & 115.2 & 115.5 & 116.6 & 117.9 & 119.8 \\
\hline \multirow{6}{*}{\multicolumn{2}{|c|}{$0.75,0.4$}} & RIDG & 61.9 & 64.1 & 62.9 & 63.1 & 62.2 & 61.9 & 64.1 & 62.9 & 63.1 & 62.2 \\
\hline & & VANILLA & 90.8 & 91.9 & 110.5 & 126.3 & 133.2 & 90.8 & 97.5 & 121.9 & 140.1 & 147.3 \\
\hline & & HIERBETAS & 51.7 & 52.2 & 53.1 & 54.3 & 55.8 & 51.7 & 52.2 & 53.1 & 54.5 & 56.1 \\
\hline & & EBBETAS & 51.8 & 52.3 & 53.2 & 54.1 & 55.2 & 51.8 & 52.3 & 53.2 & 54.2 & 55.4 \\
\hline & & EBSIGMAX & 90.8 & 109.7 & 140.0 & 152.9 & 156.1 & 90.8 & 118.6 & 152.5 & 166.5 & 169.3 \\
\hline & & ЕВВотН & 51.8 & 52.3 & 53.5 & 54.6 & 55.8 & 51.8 & 52.3 & 53.5 & 54.7 & 55.9 \\
\hline
\end{tabular}

$\operatorname{MSPE}\left(\hat{\boldsymbol{\beta}}^{p p m}\right)$ and $\operatorname{MSPE}\left(\hat{\boldsymbol{\beta}}^{\text {pm }}\right)$ under $\boldsymbol{\beta}=\left\{\{0.1\}_{k=1}^{k=8}, 1\right\}_{j=1}^{j=11}$ (a concentrated signal). When $\rho=0.15$, the error structure for $\boldsymbol{X}$ is compound symmetric, and when $\rho=0.75$, the error structure is AR(1). The column-wise minima of each box are in bold 


\begin{tabular}{|c|c|c|c|c|c|c|c|c|c|c|c|c|}
\hline \multirow[b]{2}{*}{$\rho, R^{2}$} & \multirow[b]{2}{*}{$n_{\mathrm{B}}$} & \multirow[b]{2}{*}{ Method } & \multicolumn{5}{|c|}{$\operatorname{MSPE}\left(\hat{\boldsymbol{\beta}}^{\mathrm{ppm}}\right)$} & \multicolumn{5}{|c|}{$\operatorname{MSPE}\left(\hat{\boldsymbol{\beta}}^{\mathrm{pm}}\right)$} \\
\hline & & & $\tau=0.01$ & 0.5 & 1.0 & 1.50 & 2.00 & 0.01 & 0.5 & 1.0 & 1.50 & 2.00 \\
\hline \multirow[t]{6}{*}{$0.15,0.1$} & 400 & RIDG & 74.09 & 73.55 & 73.40 & 74.01 & 74.20 & 74.09 & 73.55 & 73.40 & 74.01 & 74.20 \\
\hline & & VANILLA & 79.65 & 89.02 & 108.80 & 142.10 & 155.11 & 79.65 & 92.20 & 123.56 & 160.06 & 171.50 \\
\hline & & HIERBETAS & 67.09 & 67.72 & 68.21 & 68.36 & 68.67 & 67.09 & 67.73 & 68.22 & 68.36 & 68.64 \\
\hline & & EBBETAS & 67.99 & 68.42 & 68.66 & 68.73 & 68.77 & 67.99 & 68.42 & 68.66 & 68.73 & 68.76 \\
\hline & & EBSIGMAX & 79.66 & 88.02 & 113.97 & 147.55 & 164.04 & 79.65 & 90.89 & 129.24 & 167.26 & 180.22 \\
\hline & & ЕВВотн & 68.00 & 68.43 & 68.67 & 68.74 & 68.77 & 68.00 & 68.44 & 68.67 & 68.73 & 68.76 \\
\hline \multirow[t]{6}{*}{$0.75,0.1$} & 400 & RIDG & 523.79 & 536.20 & 519.80 & 528.23 & 532.94 & 523.79 & 536.20 & 519.80 & 528.23 & 532.94 \\
\hline & & VANILLA & 589.99 & 574.68 & 605.71 & 635.76 & 680.29 & 589.97 & 586.67 & 647.97 & 714.70 & 785.98 \\
\hline & & HIERBETAS & 471.93 & 470.84 & 472.84 & 474.42 & 480.87 & 471.92 & 470.74 & 472.56 & 473.88 & 480.37 \\
\hline & & EBBETAS & 479.69 & 477.62 & 480.63 & 482.04 & 486.65 & 479.68 & 477.55 & 480.43 & 481.60 & 486.21 \\
\hline & & EBSIGMAX & 590.06 & 620.88 & 664.24 & 735.10 & 812.12 & 590.01 & 642.94 & 731.56 & 848.85 & 952.76 \\
\hline & & ЕВВотн & 479.54 & 477.79 & 481.26 & 482.87 & 488.61 & 479.53 & 477.68 & 480.96 & 482.31 & 487.99 \\
\hline \multirow[t]{6}{*}{$0.15,0.4$} & 400 & RIDG & 17.87 & 17.54 & 17.65 & 17.46 & 17.74 & 17.87 & 17.54 & 17.65 & 17.46 & 17.74 \\
\hline & & VANILLA & 13.26 & 15.49 & 21.49 & 28.12 & 32.40 & 13.26 & 16.14 & 24.32 & 31.14 & 35.09 \\
\hline & & HIERBETAS & 12.34 & 12.93 & 13.90 & 14.74 & 15.34 & 12.34 & 12.94 & 13.93 & 14.78 & 15.39 \\
\hline & & EBBETAS & 3.44 & 13.55 & 14.41 & 15.14 & 15.74 & 13.43 & 13.56 & 14.44 & 15.17 & 15.77 \\
\hline & & EBSIGMAX & 13.26 & 15.27 & 21.91 & 30.26 & 34.17 & 13.26 & 15.85 & 24.87 & 33.37 & 36.86 \\
\hline & & ЕВВотН & 13.48 & 13.54 & 14.43 & 15.16 & 15.75 & 13.48 & 13.55 & 14.45 & 15.18 & 15.78 \\
\hline \multirow[t]{6}{*}{$0.75,0.4$} & 400 & RIDG & 100.42 & 100.89 & 99.81 & 99.95 & 101.02 & 100.42 & 100.89 & 99.81 & 99.95 & 101.02 \\
\hline & & VANILLA & 7.91 & 97.28 & 102.18 & 110.06 & 120.13 & 97.90 & 99.31 & 109.61 & 124.62 & 139.82 \\
\hline & & HIERBETAS & 81.01 & 81.69 & 81.90 & 83.74 & 86.36 & 81.01 & 81.68 & 81.90 & 83.92 & 86.79 \\
\hline & & EBBETAS & 11 & 81.78 & 81.73 & 83.65 & 86.02 & 81.11 & 81.76 & 81.72 & 83.76 & 86.27 \\
\hline & & EBSIGM & 97.92 & 105.03 & 113.59 & 128.58 & 146.11 & 97.92 & 108.85 & 125.36 & 148.88 & 170.47 \\
\hline & & ЕВВотН & 81.09 & 81.71 & 82.49 & 84.89 & 87.65 & 81.09 & 81.68 & 82.47 & 84.97 & 87.80 \\
\hline \multirow[t]{6}{*}{$0.15,0.1$} & 150 & RIDG & 73.44 & 73.80 & 74.22 & 74.13 & 73.53 & 73.44 & 73.80 & 74.22 & 74.13 & 73.53 \\
\hline & & VANILLA & 125.29 & 149.56 & 235.37 & 230.94 & 223.55 & 125.27 & 160.03 & 251.59 & 246.44 & 237.77 \\
\hline & & HIERBETAS & 68.45 & 68.81 & 69.25 & 69.50 & 69.31 & 68.45 & 68.80 & 69.20 & 69.42 & 69.26 \\
\hline & & EBBETAS & 68.77 & 69.07 & 69.30 & 69.25 & 69.17 & 68.77 & 69.07 & 69.28 & 69.23 & 69.14 \\
\hline & & EBSIGMAX & 125.21 & 150.29 & 239.49 & 240.20 & 233.70 & 125.21 & 160.60 & 256.19 & 254.68 & 248.14 \\
\hline & & ЕВвотн & 68.78 & 69.07 & 69.30 & 69.27 & 69.19 & 68.78 & 69.06 & 69.29 & 69.25 & 69.16 \\
\hline \multirow[t]{6}{*}{$0.75,0.1$} & 150 & RIDG & 528.41 & 528.12 & 542.56 & 527.94 & 529.34 & 528.41 & 528.12 & 542.56 & 527.94 & 529.34 \\
\hline & & VANILLA & 929.85 & 916.60 & 1083.86 & 1175.04 & 1196.09 & 929.57 & 971.78 & 1195.87 & 1311.07 & 1330.01 \\
\hline & & HIERBETAS & 483.01 & 483.69 & 487.68 & 491.58 & 493.62 & 483.00 & 483.50 & 487.21 & 491.14 & 493.77 \\
\hline & & EBBETAS & 489.80 & 490.23 & 493.83 & 495.78 & 499.95 & 489.80 & 490.10 & 493.46 & 495.29 & 499.75 \\
\hline & & EBSIGMAX & 929.33 & 1093.62 & 1296.55 & 1401.16 & 1402.45 & 929.36 & 1181.01 & 1423.90 & 1532.15 & 1537.75 \\
\hline & & ЕВВОтн & 489.55 & 490.39 & 494.66 & 496.66 & 501.03 & 489.54 & 490.23 & 494.21 & 496.05 & 500.73 \\
\hline \multirow{6}{*}{\multicolumn{2}{|c|}{$0.15,0.4$}} & RIDG & 17.81 & 17.73 & 17.74 & 17.65 & 17.67 & 17.81 & 17.73 & 17.74 & 17.65 & 17.67 \\
\hline & & VANILLA & 20.80 & 26.76 & 45.28 & 46.28 & 46.78 & 20.79 & 28.61 & 48.25 & 48.94 & 49.46 \\
\hline & & HIERBETAS & 14.08 & 14.49 & 15.34 & 16.16 & 16.81 & 14.08 & 14.50 & 15.37 & 16.19 & 16.84 \\
\hline & & EBBETAS & 14.72 & 14.94 & 15.71 & 16.12 & 16.46 & 14.72 & 14.94 & 15.72 & 16.13 & 16.47 \\
\hline & & EBSIGMAX & 20.79 & 26.41 & 46.27 & 47.74 & 48.51 & 20.79 & 28.22 & 49.19 & 50.48 & 51.00 \\
\hline & & ЕВВотн & 14.71 & 14.95 & 15.70 & 16.13 & 16.45 & 14.71 & 14.95 & 15.72 & 16.14 & 16.46 \\
\hline \multirow{6}{*}{\multicolumn{2}{|c|}{$0.75,0.4$}} & RIDG & 00.07 & 102.46 & 99.11 & 100.75 & 100.07 & 100.07 & 102.46 & 99.11 & 100.75 & 100.07 \\
\hline & & VANILLA & 150.97 & 155.73 & 183.32 & 212.93 & 225.54 & 150.95 & 164.84 & 203.10 & 234.98 & 246.53 \\
\hline & & HIERBETAS & 84.34 & 85.16 & 86.40 & 88.11 & 90.71 & 84.34 & 85.12 & 86.45 & 88.43 & 91.28 \\
\hline & & EBBETAS & 84.12 & 85.02 & 86.03 & 87.64 & 89.92 & 84.11 & 84.98 & 86.03 & 87.82 & 90.30 \\
\hline & & EBSIGMAX & 151.08 & 178.45 & 231.81 & 248.84 & 253.86 & 151.04 & 192.93 & 254.27 & 272.79 & 276.32 \\
\hline & & ЕВВотн & 84.11 & 85.11 & 86.72 & 88.70 & 91.11 & 84.11 & 85.04 & 86.68 & 88.76 & 91.31 \\
\hline
\end{tabular}

$\operatorname{MSPE}\left(\hat{\boldsymbol{\beta}}^{\text {ppm }}\right)$ and $\operatorname{MSPE}\left(\hat{\boldsymbol{\beta}}^{\text {pm }}\right)$ under $\boldsymbol{\beta}=\left\{\frac{j}{100}\right\}_{j=-49}^{j=49}$ (a diffuse signal). When $\rho=0.15$, the error structure for $\boldsymbol{X}$ is compound symmetric, and when $\rho=0.75$, the error structure is AR(1). The column-wise minima of each box are in bold 


\begin{tabular}{|c|c|c|c|c|c|c|c|c|c|c|c|}
\hline \multirow[b]{2}{*}{$\rho, R^{2}$} & \multirow[b]{2}{*}{ Method } & \multicolumn{5}{|c|}{$\operatorname{MSPE}\left(\hat{\boldsymbol{\beta}}^{\mathrm{ppm}}\right)$} & \multicolumn{5}{|c|}{$\operatorname{MSPE}\left(\hat{\boldsymbol{\beta}}^{\mathrm{pm}}\right)$} \\
\hline & & $\tau=0.01$ & 0.5 & 1.0 & 1.50 & 2.00 & 0.01 & 0.5 & 1.0 & 1.50 & 2.00 \\
\hline \multirow[t]{6}{*}{$0.15,0.1$} & RIDG & 694.3 & 702.3 & 695.4 & 690.8 & 695.2 & 694.3 & 702.3 & 695.4 & 690.8 & 695.2 \\
\hline & VANILLA & 803.9 & 882.1 & 1086.9 & 1385.8 & 1455.6 & 803.9 & 912.9 & 1231.5 & 1564.9 & 1615.3 \\
\hline & HIERBETAS & 641.1 & 636.8 & 641.8 & 643.6 & 649.9 & 641.1 & 636.4 & 640.6 & 641.8 & 647.7 \\
\hline & EBBETAS & 646.7 & 640.1 & 643.9 & 646.7 & 650.7 & 646.7 & 639.8 & 642.9 & 645.3 & 648.8 \\
\hline & EBSIGMAX & 804.1 & 872.2 & 1110.3 & 1481.1 & 1564.8 & 804.1 & 900.1 & 1259.3 & 1666.3 & 1724.4 \\
\hline & ЕВВотн & 646.4 & 640.1 & 643.9 & 647.1 & 651.0 & 646.4 & 639.8 & 642.9 & 645.7 & 649.1 \\
\hline \multirow[t]{6}{*}{$0.75,0.1$} & RIDG & 316.0 & 313.3 & 312.0 & 314.8 & 309.7 & 316.0 & 313.3 & 312.0 & 314.8 & 309.7 \\
\hline & VANILLA & 347.8 & 341.2 & 362.2 & 383.9 & 404.3 & 347.8 & 348.2 & 386.5 & 430.8 & 463.5 \\
\hline & HIERBETAS & 282.3 & 281.9 & 284.4 & 284.8 & 286.9 & 282.3 & 281.8 & 284.3 & 284.5 & 286.5 \\
\hline & EBBETAS & 286.0 & 285.7 & 288.9 & 287.8 & 290.0 & 286.0 & 285.6 & 288.9 & 287.5 & 289.7 \\
\hline & EBSIGMAX & 347.8 & 368.2 & 397.3 & 442.6 & 474.8 & 347.8 & 381.1 & 436.0 & 509.2 & 552.3 \\
\hline & ЕВвотн & 285.9 & 285.8 & 289.1 & 288.4 & 290.9 & 285.9 & 285.8 & 288.9 & 288.1 & 290.4 \\
\hline \multirow[t]{6}{*}{$0.15,0.4$} & RIDG & 125.9 & 127.6 & 127.1 & 127.2 & 126.6 & 125.9 & 127.6 & 127.1 & 127.2 & 126.6 \\
\hline & VANILLA & 133.9 & 148.3 & 186.0 & 236.9 & 263.9 & 133.9 & 153.6 & 210.5 & 267.1 & 291.6 \\
\hline & HIERBETAS & 111.7 & 111.8 & 114.1 & 114.8 & 117.2 & 111.7 & 111.8 & 113.9 & 114.5 & 116.9 \\
\hline & EBBETAS & 111.8 & 111.9 & 113.7 & 114.2 & 116.0 & 111.8 & 111.8 & 113.4 & 113.9 & 115.6 \\
\hline & EBSIGMAX & 133.9 & 146.5 & 190.4 & 248.2 & 274.5 & 133.9 & 151.3 & 216.1 & 280.2 & 303.9 \\
\hline & ЕВВОтн & 111.8 & 111.8 & 113.6 & 114.4 & 116.1 & 111.8 & 111.8 & 113.4 & 114.1 & 115.7 \\
\hline \multirow[t]{6}{*}{$0.75,0.4$} & RIDG & 62.1 & 63.0 & 62.9 & 64.1 & 61.5 & 62.1 & 63.0 & 62.9 & 64.1 & 61.5 \\
\hline & VANILLA & 58.3 & 58.2 & 62.3 & 69.1 & 72.6 & 58.3 & 59.4 & 66.8 & 77.8 & 83.8 \\
\hline & HIERBETAS & 49.3 & 49.9 & 50.6 & 51.8 & 53.1 & 49.3 & 49.9 & 50.6 & 51.9 & 53.3 \\
\hline & EBBETAS & 49.4 & 50.4 & 50.6 & 51.9 & 53.1 & 49.4 & 50.4 & 50.6 & 51.9 & 53.2 \\
\hline & EBSIGMAX & 58.3 & 62.7 & 69.1 & 80.2 & 88.6 & 58.3 & 65.0 & 76.4 & 92.2 & 102.6 \\
\hline & ЕВВОТН & 49.4 & 50.2 & 51.0 & 52.5 & 54.0 & 49.4 & 50.2 & 50.9 & 52.5 & 54.0 \\
\hline \multirow[t]{6}{*}{$0.15,0.1$} & RIDG & 686.3 & 690.7 & 691.4 & 700.9 & 689.7 & 686.3 & 690.7 & 691.4 & 700.9 & 689.7 \\
\hline & VANILLA & 1264.1 & 1494.0 & 2337.3 & 2312.8 & 2169.8 & 1264.1 & 1597.8 & 2505.8 & 2442.4 & 2318.4 \\
\hline & HIERBETAS & 646.0 & 651.4 & 649.9 & 655.7 & 658.5 & 646.0 & 650.9 & 648.5 & 653.9 & 656.2 \\
\hline & EBBETAS & 649.8 & 654.3 & 653.1 & 656.3 & 654.4 & 649.8 & 653.9 & 652.1 & 655.0 & 652.5 \\
\hline & EBSIGMAX & 1263.9 & 1490.5 & 2392.9 & 2287.6 & 2301.3 & 1263.9 & 1592.5 & 2555.5 & 2436.8 & 2438.5 \\
\hline & ЕВВОтн & 650.3 & 654.2 & 653.6 & 656.4 & 654.8 & 650.3 & 653.7 & 652.6 & 655.1 & 652.9 \\
\hline \multirow[t]{6}{*}{$0.75,0.1$} & RIDG & 315.8 & 321.2 & 311.8 & 314.2 & 316.5 & 315.8 & 321.2 & 311.8 & 314.2 & 316.5 \\
\hline & VANILLA & 546.8 & 540.1 & 635.4 & 676.1 & 707.2 & 546.8 & 573.7 & 701.9 & 753.7 & 787.1 \\
\hline & HIERBETAS & 286.9 & 288.1 & 291.0 & 295.2 & 295.3 & 286.9 & 288.0 & 290.8 & 294.8 & 295.4 \\
\hline & EBBETAS & 290.5 & 291.7 & 293.8 & 297.6 & 296.8 & 290.5 & 291.6 & 293.6 & 297.4 & 296.7 \\
\hline & EBSIGMAX & 546.6 & 649.8 & 768.3 & 811.5 & 832.4 & 546.6 & 702.5 & 845.9 & 892.2 & 918.4 \\
\hline & ЕВВОТн & 290.5 & 291.7 & 294.1 & 298.3 & 297.3 & 290.5 & 291.6 & 293.9 & 298.0 & 297.2 \\
\hline \multirow[t]{6}{*}{$0.15,0.4$} & RIDG & 127.9 & 125.2 & 124.7 & 124.8 & 124.1 & 127.9 & 125.2 & 124.7 & 124.8 & 124.1 \\
\hline & VANILLA & 208.3 & 256.8 & 403.2 & 397.2 & 391.9 & 208.3 & 275.3 & 432.3 & 422.9 & 417.6 \\
\hline & HIERBETAS & 115.3 & 115.6 & 117.2 & 119.4 & 124.5 & 115.3 & 115.5 & 116.9 & 119.0 & 124.0 \\
\hline & EBBETAS & 114.9 & 115.1 & 116.0 & 117.3 & 120.3 & 114.9 & 114.9 & 115.7 & 116.9 & 119.6 \\
\hline & EBSIGMAX & 208.2 & 253.7 & 414.9 & 428.2 & 407.8 & 208.2 & 271.7 & 443.1 & 449.7 & 433.0 \\
\hline & ЕВВОтн & 114.9 & 115.0 & 116.1 & 117.5 & 120.2 & 114.9 & 114.9 & 115.8 & 117.0 & 119.5 \\
\hline \multirow[t]{6}{*}{$0.75,0.4$} & RIDG & 63.5 & 62.5 & 62.7 & 63.3 & 61.6 & 63.5 & 62.5 & 62.7 & 63.3 & 61.6 \\
\hline & VANILLA & 90.6 & 91.0 & 111.7 & 127.7 & 132.5 & 90.6 & 96.6 & 123.4 & 140.3 & 146.1 \\
\hline & HIERBETAS & 51.5 & 52.2 & 53.3 & 54.8 & 56.2 & 51.5 & 52.1 & 53.4 & 54.9 & 56.4 \\
\hline & EBBETAS & 51.6 & 52.4 & 53.3 & 54.4 & 55.8 & 51.6 & 52.3 & 53.3 & 54.5 & 55.9 \\
\hline & EBSIGMAX & 90.7 & 111.6 & 141.2 & 145.9 & 155.7 & 90.6 & 120.3 & 155.3 & 160.1 & 168.9 \\
\hline & ЕВВотн & 51.6 & 52.4 & 53.5 & 54.9 & 56.4 & 51.6 & 52.4 & 53.5 & 54.9 & 56.4 \\
\hline
\end{tabular}

$\operatorname{MSPE}\left(\hat{\boldsymbol{\beta}}^{\text {ppm }}\right)$ and $\operatorname{MSPE}\left(\hat{\boldsymbol{\beta}}^{\text {pm }}\right)$ under $\boldsymbol{\beta}=\left\{\{0.1\}_{k=1}^{k=8}, 1\right\}_{j=1}^{j=11}$ (a concentrated signal) when $\varepsilon+1 \sim G(1,1)$. When $\rho=0.15$, the error structure for $\boldsymbol{X}$ is compound symmetric, and when $\rho=0.75$, the error structure is AR(1). The column-wise minima of each box are in bold 


\begin{tabular}{|c|c|c|c|c|c|c|c|c|c|c|c|}
\hline \multirow[b]{2}{*}{$\rho, R^{2}$} & \multirow[b]{2}{*}{ Method } & \multicolumn{5}{|c|}{$\operatorname{MSPE}\left(\hat{\boldsymbol{\beta}}^{\mathrm{ppm}}\right)$} & \multicolumn{5}{|c|}{$\operatorname{MSPE}\left(\hat{\boldsymbol{\beta}}^{\mathrm{pm}}\right)$} \\
\hline & & $\tau=0.01$ & 0.5 & 1.0 & 1.50 & 2.00 & 0.01 & 0.5 & 1.0 & 1.50 & 2.00 \\
\hline \multirow[t]{6}{*}{$0.15,0.1$} & RIDG & 73.84 & 73.22 & 73.73 & 73.77 & 74.22 & 73.84 & 73.22 & 73.73 & 73.77 & 74.22 \\
\hline & VANILLA & 79.61 & 88.78 & 109.85 & 139.23 & 147.96 & 79.61 & 91.95 & 124.40 & 157.32 & 164.91 \\
\hline & HIERBETAS & 67.27 & 67.35 & 68.41 & 68.36 & 68.85 & 67.27 & 67.35 & 68.40 & 68.35 & 68.82 \\
\hline & EBBETAS & 68.13 & 68.02 & 68.84 & 68.63 & 69.00 & 68.13 & 68.02 & 68.85 & 68.62 & 68.99 \\
\hline & EBSIGMAX & 79.62 & 87.78 & 111.84 & 146.90 & 161.51 & 79.62 & 90.65 & 127.13 & 165.78 & 178.25 \\
\hline & Еввотн & 68.11 & 68.01 & 68.84 & 68.62 & 69.01 & 68.11 & 68.01 & 68.84 & 68.61 & 69.00 \\
\hline \multirow[t]{6}{*}{$0.75,0.1$} & RIDG & 525.97 & 519.50 & 529.63 & 529.43 & 539.07 & 525.97 & 519.50 & 529.63 & 529.43 & 539.07 \\
\hline & VANILLA & 587.50 & 577.27 & 607.07 & 649.61 & 688.95 & 587.47 & 589.35 & 649.28 & 728.16 & 792.52 \\
\hline & HIERBETAS & 471.30 & 471.74 & 473.28 & 477.64 & 483.86 & 471.29 & 471.66 & 473.01 & 477.14 & 483.17 \\
\hline & EBBETAS & 479.13 & 478.80 & 481.39 & 484.81 & 490.66 & 479.13 & 478.74 & 481.19 & 484.38 & 490.16 \\
\hline & EBSIGMAX & 587.59 & 624.21 & 665.89 & 737.95 & 824.74 & 587.58 & 646.43 & 731.43 & 847.48 & 955.73 \\
\hline & ЕВВОТН & 478.90 & 478.88 & 481.92 & 485.81 & 492.33 & 478.89 & 478.79 & 481.64 & 485.27 & 491.71 \\
\hline \multirow[t]{6}{*}{$0.15,0.4$} & RIDG & 17.52 & 17.48 & 17.79 & 17.60 & 17.82 & 17.52 & 17.48 & 17.79 & 17.60 & 17.82 \\
\hline & VANILLA & 13.25 & 15.31 & 21.20 & 29.35 & 31.43 & 13.25 & 15.95 & 23.96 & 32.21 & 34.35 \\
\hline & HIERBETAS & 12.31 & 12.86 & 13.81 & 14.80 & 15.48 & 12.31 & 12.87 & 13.84 & 14.84 & 15.54 \\
\hline & EBBETAS & 13.24 & 13.67 & 14.48 & 15.27 & 15.72 & 13.24 & 13.68 & 14.51 & 15.29 & 15.75 \\
\hline & EBSIGMAX & 13.25 & 15.12 & 21.55 & 31.23 & 32.95 & 13.25 & 15.68 & 24.52 & 34.16 & 35.79 \\
\hline & ЕВВОТН & 13.26 & 13.65 & 14.51 & 15.27 & 15.73 & 13.26 & 13.65 & 14.53 & 15.30 & 15.76 \\
\hline \multirow[t]{6}{*}{$0.75,0.4$} & RIDG & 100.36 & 99.95 & 102.19 & 101.45 & 100.97 & 100.36 & 99.95 & 102.19 & 101.45 & 100.97 \\
\hline & VANILLA & 6.98 & 97.10 & 103.06 & 111.51 & 121.80 & 96.98 & 99.11 & 110.53 & 126.37 & 141.21 \\
\hline & HIERBETAS & 80.47 & 81.55 & 82.44 & 83.77 & 87.21 & 80.47 & 81.53 & 82.45 & 83.93 & 87.60 \\
\hline & EBBETAS & 80.76 & 81.81 & 82.44 & 83.39 & 86.66 & 80.76 & 81.79 & 82.43 & 83.48 & 86.90 \\
\hline & EBSIGMAX & 7.01 & 104.86 & 113.87 & 129.09 & 150.33 & 97.01 & 108.63 & 125.65 & 150.12 & 174.52 \\
\hline & ЕВВОТН & 80.76 & 81.82 & 83.08 & 84.63 & 88.16 & 80.75 & 81.79 & 83.06 & 84.67 & 88.21 \\
\hline \multirow[t]{6}{*}{$0.15,0.1$} & RIDG & 74.00 & 74.49 & 73.96 & 73.94 & 73.07 & 74.00 & 74.49 & 73.96 & 73.94 & 73.07 \\
\hline & VANILLA & 124.35 & 152.68 & 231.58 & 235.34 & 229.21 & 124.34 & 163.65 & 248.06 & 249.57 & 244.11 \\
\hline & HIERBETAS & 68.69 & 68.37 & 69.11 & 69.25 & 69.62 & 68.68 & 68.36 & 69.07 & 69.20 & 69.54 \\
\hline & EBBETAS & 9.11 & 68.69 & 69.17 & 69.30 & 69.11 & 69.12 & 68.69 & 69.15 & 69.28 & 69.08 \\
\hline & EBSIGMAX & 124.36 & 151.44 & 244.88 & 236.04 & 244.40 & 124.35 & 162.07 & 260.73 & 251.66 & 259.36 \\
\hline & ЕВВОтН & 69.12 & 68.70 & 69.18 & 69.32 & 69.11 & 69.12 & 68.69 & 69.17 & 69.31 & 69.07 \\
\hline \multirow[t]{6}{*}{$0.75,0.1$} & RIDG & 534.02 & 527.23 & 531.07 & 523.91 & 526.81 & 534.02 & 527.23 & 531.07 & 523.91 & 526.81 \\
\hline & VANILLA & 915.42 & 901.20 & 1074.68 & 1175.88 & 1133.98 & 915.29 & 957.72 & 1190.97 & 1305.25 & 1277.79 \\
\hline & HIERBETAS & 480.51 & 483.15 & 485.01 & 487.88 & 497.03 & 480.52 & 483.00 & 484.54 & 487.29 & 496.75 \\
\hline & EBBETAS & 486.68 & 489.21 & 491.33 & 492.71 & 499.40 & 486.69 & 489.09 & 490.93 & 492.08 & 498.76 \\
\hline & EBSIGMAX & 915.69 & 1084.99 & 1325.31 & 1360.39 & 1423.70 & 915.61 & 1172.33 & 1463.78 & 1492.13 & 1567.57 \\
\hline & ЕВВОТн & 486.58 & 489.73 & 491.92 & 493.80 & 500.29 & 486.59 & 489.60 & 491.45 & 493.14 & 499.71 \\
\hline \multirow[t]{6}{*}{$0.15,0.4$} & RIDG & 17.53 & 17.55 & 17.41 & 17.74 & 17.48 & 17.53 & 17.55 & 17.41 & 17.74 & 17.48 \\
\hline & VANILLA & 20.63 & 26.37 & 45.47 & 46.16 & 47.40 & 20.63 & 28.27 & 48.29 & 48.86 & 49.99 \\
\hline & HIERBETAS & 14.11 & 14.63 & 15.35 & 16.24 & 16.64 & 14.11 & 14.64 & 15.38 & 16.28 & 16.67 \\
\hline & EBBETAS & 14.70 & 15.11 & 15.69 & 16.18 & 16.35 & 14.70 & 15.11 & 15.70 & 16.19 & 16.34 \\
\hline & EBSIGMAX & 20.64 & 26.38 & 45.27 & 47.65 & 47.16 & 20.63 & 28.24 & 48.35 & 50.36 & 50.26 \\
\hline & ЕВВОтн & 14.69 & 15.09 & 15.68 & 16.20 & 16.37 & 14.69 & 15.10 & 15.69 & 16.21 & 16.37 \\
\hline \multirow[t]{6}{*}{$0.75,0.4$} & RIDG & 100.53 & 103.75 & 100.36 & 100.05 & 101.42 & 100.53 & 103.75 & 100.36 & 100.05 & 101.42 \\
\hline & VANILLA & 154.31 & 150.90 & 180.92 & 208.60 & 216.98 & 154.28 & 160.27 & 200.19 & 231.10 & 239.66 \\
\hline & HIERBETAS & 84.55 & 85.61 & 86.85 & 88.51 & 90.85 & 84.55 & 85.59 & 86.84 & 88.79 & 91.42 \\
\hline & EBBETAS & 84.66 & 85.44 & 86.47 & 87.93 & 90.17 & 84.65 & 85.41 & 86.42 & 88.06 & 90.54 \\
\hline & EBSIGMAX & 154.36 & 184.58 & 232.36 & 239.26 & 252.38 & 154.34 & 199.06 & 253.12 & 263.95 & 277.45 \\
\hline & ЕВВОTH & 84.65 & 85.45 & 86.88 & 88.92 & 91.44 & 84.64 & 85.39 & 86.77 & 88.98 & 91.64 \\
\hline
\end{tabular}

$\operatorname{MSPE}\left(\hat{\boldsymbol{\beta}}^{\text {ppm }}\right)$ and $\operatorname{MSPE}\left(\hat{\boldsymbol{\beta}}^{\text {pm }}\right)$ under $\boldsymbol{\beta}=\left\{\frac{j}{100}\right\}_{j=-49}^{j=49}$ (a diffuse signal) when $\varepsilon+1 \sim G(1,1)$. When $\rho=0.15$, the error structure for $\boldsymbol{X}$ is compound symmetric, and when $\rho=0.75$, the error structure is AR(1). The column-wise minima of each box are in bold 


\begin{tabular}{|c|c|c|c|c|c|c|c|c|c|c|c|c|}
\hline \multirow[b]{2}{*}{$\rho, R^{2}$} & \multirow[b]{2}{*}{$n_{\mathrm{B}}$} & \multirow[b]{2}{*}{ Method } & \multicolumn{5}{|c|}{$\operatorname{MSPE}\left(\hat{\boldsymbol{\beta}}^{\mathrm{ppm}}\right)$} & \multicolumn{5}{|c|}{$\operatorname{MSPE}\left(\hat{\boldsymbol{\beta}}^{\mathrm{pm}}\right)$} \\
\hline & & & $\tau=0.01$ & 0.5 & 1.0 & 1.50 & 2.00 & 0.01 & 0.5 & 1.0 & 1.50 & 2.00 \\
\hline \multirow{6}{*}{$0.15,0.1$} & 400 & RIDG & 689.0 & 693.5 & 703.6 & 696.7 & 710.2 & 689.0 & 693.5 & 703.6 & 696.7 & 710.2 \\
\hline & & VANILLA & 864.9 & 899.7 & 971.6 & 1093.5 & 1266.1 & 884.6 & 935.1 & 1054.5 & 1244.6 & 1452.6 \\
\hline & & HIERBETAS & 638.0 & 638.6 & 637.4 & 641.1 & 644.5 & 637.7 & 638.3 & 636.7 & 639.9 & 643.2 \\
\hline & & EBBETAS & 643.5 & 644.5 & 643.6 & 645.8 & 650.0 & 643.3 & 644.2 & 643.0 & 644.9 & 649.0 \\
\hline & & EBSIGMAX & 856.0 & 894.8 & 972.3 & 1120.2 & 1330.5 & 874.4 & 928.9 & 1053.9 & 1277.7 & 1511.8 \\
\hline & & ЕВВОТН & 643.6 & 644.1 & 643.4 & 645.5 & 649.8 & 643.5 & 643.9 & 642.9 & 644.6 & 648.9 \\
\hline \multirow[t]{6}{*}{$\overline{0.75,0.1}$} & 400 & RIDG & 316.4 & 316.6 & 317.2 & 315.1 & 315.2 & 316.4 & 316.6 & 317.2 & 315.1 & 315.2 \\
\hline & & VANILLA & 348.8 & 348.2 & 350.0 & 358.4 & 372.5 & 354.7 & 356.1 & 364.1 & 382.7 & 410.9 \\
\hline & & HIERBETAS & 282.9 & 283.3 & 282.8 & 282.6 & 284.5 & 282.9 & 283.3 & 282.8 & 282.5 & 284.2 \\
\hline & & EBBETAS & 287.4 & 288.5 & 287.6 & 286.8 & 288.7 & 287.4 & 288.5 & 287.5 & 286.7 & 288.5 \\
\hline & & EBSIGMAX & 382.6 & 382.8 & 384.2 & 394.9 & 415.0 & 395.0 & 398.6 & 409.5 & 433.8 & 471.9 \\
\hline & & ЕВвотн & 287.4 & 288.2 & 287.5 & 287.1 & 289.0 & 287.4 & 288.2 & 287.5 & 287.0 & 288.7 \\
\hline \multirow[t]{6}{*}{$0.15,0.4$} & 400 & RIDG & 125.6 & 126.4 & 126.4 & 126.4 & 126.5 & 125.6 & 126.4 & 126.4 & 126.4 & 126.5 \\
\hline & & VANILLA & 146.1 & 150.6 & 165.4 & 191.4 & 219.8 & 149.6 & 156.8 & 179.8 & 216.8 & 249.2 \\
\hline & & HIERBETAS & 112.2 & 111.5 & 113.3 & 114.0 & 114.1 & 112.2 & 111.5 & 113.2 & 113.8 & 113.8 \\
\hline & & EBBETAS & 112.0 & 111.3 & 113.0 & 113.6 & 113.5 & 112.0 & 111.3 & 112.8 & 113.4 & 113.2 \\
\hline & & EBSIGMAX & 144.6 & 149.6 & 166.0 & 193.2 & 233.9 & 147.8 & 155.4 & 180.3 & 220.0 & 265.6 \\
\hline & & ЕВвотн & 112.0 & 111.3 & 112.9 & 113.5 & 113.5 & 112.0 & 111.2 & 112.8 & 113.3 & 113.2 \\
\hline \multirow[t]{6}{*}{$0.75,0.4$} & 400 & RIDG & 62.2 & 61.0 & 61.0 & 62.6 & 61.5 & 62.2 & 61.0 & 61.0 & 62.6 & 61.5 \\
\hline & & VANILLA & 59.3 & 58.8 & 59.6 & 61.8 & 65.1 & 60.3 & 60.1 & 62.0 & 66.3 & 72.3 \\
\hline & & HIERBETAS & 50.1 & 49.8 & 50.0 & 50.2 & 50.9 & 50.1 & 49.8 & 50.0 & 50.2 & 51.0 \\
\hline & & EBBETAS & 50.1 & 49.9 & 50.2 & 50.1 & 50.9 & 50.1 & 49.9 & 50.2 & 50.1 & 50.9 \\
\hline & & EBSIGMAX & 65.0 & 64.4 & 65.6 & 68.4 & 73.3 & 67.1 & 67.2 & 70.1 & 75.9 & 83.9 \\
\hline & & ЕВвотн & 49.8 & 49.6 & 50.1 & 50.3 & 51.3 & 49.8 & 49.6 & 50.1 & 50.3 & 51.3 \\
\hline \multirow[t]{6}{*}{$0.15,0.1$} & 150 & RIDG & 700.8 & 692.6 & 696.9 & 695.6 & 685.1 & 700.8 & 692.6 & 696.9 & 695.6 & 685.1 \\
\hline & & VANILLA & 1448.2 & 1553.3 & 2123.5 & 2377.5 & 2272.9 & 1522.3 & 1671.9 & 2309.3 & 2541.8 & 2427.6 \\
\hline & & HIERBETAS & 649.3 & 648.6 & 652.0 & 653.9 & 653.1 & 649.0 & 648.1 & 651.1 & 652.5 & 651.4 \\
\hline & & EBBETAS & 655.4 & 653.2 & 656.8 & 658.2 & 656.5 & 655.1 & 652.7 & 656.2 & 657.1 & 655.3 \\
\hline & & EBSIGMAX & 1449.1 & 1546.1 & 2173.8 & 2370.7 & 2329.3 & 1525.3 & 1667.1 & 2368.1 & 2551.9 & 2492.8 \\
\hline & & ЕВВотн & 655.4 & 653.0 & 656.8 & 657.4 & 656.6 & 655.1 & 652.6 & 656.2 & 656.4 & 655.4 \\
\hline \multirow[t]{6}{*}{$0.75,0.1$} & 150 & RIDG & 320.9 & 314.4 & 314.4 & 317.1 & 313.1 & 320.9 & 314.4 & 314.4 & 317.1 & 313.1 \\
\hline & & VANILLA & 572.4 & 570.4 & 601.9 & 647.3 & 660.5 & 600.2 & 607.2 & 656.6 & 718.3 & 741.9 \\
\hline & & HIERBETAS & 289.5 & 288.6 & 288.7 & 290.7 & 291.3 & 289.4 & 288.5 & 288.6 & 290.5 & 291.2 \\
\hline & & EBBETAS & 293.0 & 292.0 & 291.7 & 293.3 & 294.3 & 293.0 & 291.9 & 291.6 & 293.1 & 294.1 \\
\hline & & EBSIGMAX & 703.6 & 730.9 & 768.0 & 841.4 & 832.0 & 752.0 & 786.7 & 837.2 & 914.8 & 909.2 \\
\hline & & ЕВвОтн & 293.1 & 292.2 & 292.0 & 293.6 & 294.8 & 293.1 & 292.2 & 291.8 & 293.4 & 294.6 \\
\hline \multirow{6}{*}{\multicolumn{2}{|c|}{$0.15,0.4$}} & RIDG & 126.1 & 125.8 & 126.4 & 125.8 & 124.4 & 126.1 & 125.8 & 126.4 & 125.8 & 124.4 \\
\hline & & VANILLA & 242.1 & 260.7 & 372.3 & 409.2 & 401.1 & 254.7 & 280.6 & 401.2 & 434.6 & 423.9 \\
\hline & & HIERBETAS & 115.3 & 115.9 & 116.4 & 117.3 & 119.1 & 115.3 & 115.7 & 116.1 & 117.0 & 118.7 \\
\hline & & EBBETAS & 114.9 & 115.3 & 115.5 & 116.3 & 117.5 & 114.8 & 115.1 & 115.3 & 115.9 & 117.1 \\
\hline & & EBSIGMAX & 242.4 & 261.4 & 376.0 & 415.3 & 408.5 & 255.3 & 281.7 & 405.8 & 442.2 & 433.9 \\
\hline & & ЕВвОтн & 114.8 & 115.2 & 115.4 & 116.3 & 117.5 & 114.7 & 115.1 & 115.2 & 116.0 & 117.1 \\
\hline \multirow{6}{*}{\multicolumn{2}{|c|}{$0.75,0.4 \quad 150$}} & RIDG & 62.0 & 63.4 & 62.1 & 62.6 & 61.9 & 62.0 & 63.4 & 62.1 & 62.6 & 61.9 \\
\hline & & VANILLA & 98.4 & 100.0 & 106.0 & 116.0 & 125.1 & 103.2 & 106.4 & 115.5 & 127.6 & 138.0 \\
\hline & & HIERBETAS & 52.1 & 52.4 & 52.6 & 53.0 & 54.0 & 52.0 & 52.4 & 52.6 & 53.1 & 54.1 \\
\hline & & EBBETAS & 52.0 & 52.5 & 52.6 & 52.9 & 53.7 & 52.0 & 52.5 & 52.5 & 52.9 & 53.7 \\
\hline & & EBSIGMAX & 119.3 & 124.5 & 132.6 & 140.9 & 147.9 & 127.8 & 134.6 & 145.0 & 153.4 & 161.5 \\
\hline & & ЕВВОТн & 52.0 & 52.4 & 52.7 & 53.2 & 54.2 & 52.0 & 52.4 & 52.6 & 53.2 & 54.2 \\
\hline
\end{tabular}

$\boldsymbol{W} \mid \boldsymbol{X} \sim N_{p}\left(\psi \mathbf{1}_{p}+\nu \boldsymbol{X}^{2}, \tau^{2} \boldsymbol{I}_{p}\right)$, where $\boldsymbol{X}^{2}$ indicates the element-wise square of $\boldsymbol{X}$. When $\rho=0.15$, the error structure for $\boldsymbol{X}$ is compound symmetric, and when $\rho=0.75$, the error structure is AR(1). The column-wise minima of each box are in bold 


\begin{tabular}{|c|c|c|c|c|c|c|c|c|c|c|c|c|}
\hline \multirow[b]{2}{*}{$\rho, R^{2}$} & \multirow[b]{2}{*}{$n_{\mathrm{B}}$} & \multirow[b]{2}{*}{ Method } & \multicolumn{5}{|c|}{$\operatorname{MSPE}\left(\hat{\boldsymbol{\beta}}^{\mathrm{ppm}}\right)$} & \multicolumn{5}{|c|}{$\operatorname{MSPE}\left(\hat{\boldsymbol{\beta}}^{\mathrm{pm}}\right)$} \\
\hline & & & $\tau=0.01$ & 0.5 & 1.0 & 1.50 & 2.00 & 0.01 & 0.5 & 1.0 & 1.50 & 2.00 \\
\hline \multirow[t]{6}{*}{$0.15,0.1$} & 400 & RIDG & 75.10 & 72.25 & 73.91 & 74.15 & 73.60 & 75.10 & 72.25 & 73.91 & 74.15 & 73.60 \\
\hline & & VANILLA & 87.54 & 89.92 & 98.18 & 112.78 & 129.54 & 89.67 & 93.61 & 106.70 & 128.09 & 147.15 \\
\hline & & HIERBETAS & 67.43 & 67.29 & 67.72 & 68.04 & 68.43 & 67.43 & 67.31 & 67.73 & 68.06 & 68.42 \\
\hline & & EBBETAS & 68.14 & 68.02 & 68.18 & 68.51 & 68.73 & 68.14 & 68.03 & 68.18 & 68.51 & 68.73 \\
\hline & & EBSIGMAX & 86.73 & 89.20 & 98.39 & 114.34 & 134.22 & 88.73 & 92.68 & 106.81 & 130.13 & 153.70 \\
\hline & & ЕВВотн & 68.12 & 68.01 & 68.20 & 68.52 & 68.73 & 68.12 & 68.02 & 68.20 & 68.53 & 68.73 \\
\hline \multirow[t]{6}{*}{$0.75,0.1$} & 400 & RIDG & 524.29 & 528.26 & 531.41 & 541.77 & 529.11 & 524.29 & 528.26 & 531.41 & 541.77 & 529.11 \\
\hline & & VANILLA & 584.04 & 583.72 & 592.24 & 602.77 & 619.55 & 593.97 & 597.31 & 616.48 & 644.38 & 682.53 \\
\hline & & HIERBETAS & 472.23 & 472.68 & 474.51 & 472.27 & 475.34 & 472.18 & 472.62 & 474.34 & 472.03 & 474.99 \\
\hline & & EBBETAS & 479.90 & 481.08 & 480.97 & 479.17 & 482.72 & 479.86 & 481.03 & 480.84 & 478.97 & 482.45 \\
\hline & & EBSIGMAX & 639.99 & 641.80 & 650.82 & 661.56 & 693.07 & 660.47 & 668.71 & 694.08 & 728.55 & 787.97 \\
\hline & & ЕВВотн & 479.45 & 481.21 & 481.50 & 479.55 & 483.35 & 479.40 & 481.14 & 481.30 & 479.26 & 482.99 \\
\hline \multirow[t]{6}{*}{$0.15,0.4$} & 400 & RIDG & 17.65 & 17.88 & 17.69 & 17.63 & 17.72 & 17.65 & 17.88 & 17.69 & 17.63 & 17.72 \\
\hline & & VANILLA & 15.01 & 15.63 & 17.74 & 21.95 & 26.74 & 15.42 & 16.36 & 19.50 & 24.75 & 29.87 \\
\hline & & HIERBETAS & 12.82 & 12.96 & 13.41 & 13.97 & 14.48 & 12.84 & 12.97 & 13.43 & 14.00 & 14.52 \\
\hline & & EBBETAS & 13.81 & 13.90 & 14.18 & 14.55 & 15.08 & 13.82 & 13.91 & 14.20 & 14.57 & 15.11 \\
\hline & & EBSIGMAX & 14.87 & 15.49 & 17.73 & 22.44 & 27.77 & 15.25 & 16.16 & 19.47 & 25.40 & 31.01 \\
\hline & & ЕВВотн & 13.81 & 13.89 & 14.17 & 14.56 & 15.10 & 13.81 & 13.90 & 14.19 & 14.58 & 15.13 \\
\hline \multirow[t]{6}{*}{$0.75,0.4$} & 400 & RIDG & 101.25 & 101.28 & 101.29 & 100.41 & 102.31 & 101.25 & 101.28 & 101.29 & 100.41 & 102.31 \\
\hline & & VANILLA & 99.75 & 98.48 & 99.48 & 102.85 & 107.06 & 101.45 & 100.73 & 103.54 & 110.50 & 119.08 \\
\hline & & HIERBETAS & 82.30 & 81.98 & 82.12 & 82.10 & 82.48 & 82.28 & 81.97 & 82.09 & 82.09 & 82.54 \\
\hline & & EBBETAS & 82.21 & 82.08 & 82.01 & 82.12 & 82.61 & 82.19 & 82.06 & 81.98 & 82.09 & 82.62 \\
\hline & & EBSIGMAX & 109.15 & 108.12 & 109.00 & 113.94 & 120.77 & 112.76 & 112.74 & 116.36 & 126.31 & 138.51 \\
\hline & & ЕВВотн & 81.64 & 81.70 & 81.90 & 82.54 & 83.35 & 81.61 & 81.67 & 81.86 & 82.50 & 83.36 \\
\hline \multirow[t]{6}{*}{$0.15,0.1$} & 150 & RIDG & 73.50 & 73.49 & 74.30 & 73.21 & 74.63 & 73.50 & 73.49 & 74.30 & 73.21 & 74.63 \\
\hline & & VANILLA & 145.41 & 156.82 & 219.88 & 247.75 & 228.21 & 153.00 & 168.59 & 238.20 & 263.22 & 244.01 \\
\hline & & HIERBETAS & 68.84 & 68.67 & 68.82 & 69.22 & 69.12 & 68.84 & 68.66 & 68.80 & 69.18 & 69.08 \\
\hline & & EBBETAS & 69.21 & 68.92 & 69.00 & 69.35 & 69.07 & 69.20 & 68.92 & 68.99 & 69.33 & 69.05 \\
\hline & & EBSIGMAX & 145.10 & 156.57 & 219.95 & 243.12 & 241.33 & 152.73 & 168.72 & 239.52 & 259.69 & 256.94 \\
\hline & & ЕВВотн & 69.19 & 68.92 & 69.01 & 69.35 & 69.09 & 69.19 & 68.91 & 69.00 & 69.33 & 69.07 \\
\hline \multirow[t]{6}{*}{$0.75,0.1$} & 150 & RIDG & 528.96 & 529.17 & 530.14 & 528.99 & 530.43 & 528.96 & 529.17 & 530.14 & 528.99 & 530.43 \\
\hline & & VANILLA & 962.97 & 965.57 & 994.39 & 1063.71 & 1135.82 & 1010.05 & 1030.88 & 1083.29 & 1181.47 & 1260.04 \\
\hline & & HIERBETAS & 483.81 & 483.32 & 483.71 & 486.35 & 484.78 & 483.71 & 483.16 & 483.49 & 486.05 & 484.40 \\
\hline & & EBBETAS & & 488.99 & 489.08 & 493.13 & 8.99 & 89.32 & 488.87 & 488.89 & 492.88 & 488.69 \\
\hline & & EBSIGMAX & 1154.89 & 1201.38 & 1249.35 & 1318.50 & 1328.89 & 1236.38 & 1304.62 & 1369.75 & 1452.56 & 1466.23 \\
\hline & & ЕВВотн & 9.38 & 489.34 & 489.36 & 493.67 & 489.76 & 489.28 & 489.19 & 489.12 & 493.39 & 489.42 \\
\hline \multirow[t]{6}{*}{$0.15,0.4$} & 150 & RIDG & 17.43 & 17.61 & 17.64 & 17.62 & 17.57 & 17.43 & 17.61 & 17.64 & 17.62 & 17.57 \\
\hline & & VANILLA & 25.41 & 28.14 & 42.26 & 45.29 & 45.49 & 26.78 & 30.27 & 45.33 & 48.09 & 48.28 \\
\hline & & HIERBETAS & 14.35 & 14.63 & 14.99 & 15.34 & 15.97 & 14.36 & 14.64 & 15.01 & 15.38 & 16.01 \\
\hline & & EBBETAS & 14.94 & 15.19 & 15.40 & 15.62 & 15.99 & 14.94 & 15.20 & 15.41 & 15.64 & 16.01 \\
\hline & & EBSIGMAX & 25.42 & 28.51 & 41.43 & 46.08 & 46.27 & 26.83 & 30.71 & 44.80 & 49.03 & 49.09 \\
\hline & & ЕВВотн & 14.94 & 15.20 & 15.42 & 15.61 & 15.99 & 14.94 & 15.21 & 15.43 & 15.63 & 16.01 \\
\hline \multirow[t]{6}{*}{$0.75,0.4$} & 150 & RIDG & 1.14 & 100.77 & 100.16 & 101.35 & 99.94 & 101.14 & 100.77 & 100.16 & 101.35 & 99.94 \\
\hline & & VANILLA & & 160.10 & 176.08 & 187.37 & 201.27 & 171.37 & 170.75 & 192.54 & 206.59 & 222.86 \\
\hline & & HIERBETAS & 5.18 & 85.38 & 85.72 & 86.57 & 87.69 & 85.15 & 85.32 & 85.68 & 86.61 & 87.94 \\
\hline & & EBBETAS & 84.92 & 85.07 & 85.50 & 86.53 & 86.99 & 84.88 & 85.01 & 85.44 & 86.52 & 87.13 \\
\hline & & EBSIGMAX & 203.78 & 204.22 & 218.22 & 227.21 & 239.17 & 217.37 & 221.42 & 239.34 & 249.38 & 263.00 \\
\hline & & ЕВВотн & 84.86 & 85.15 & 85.70 & 86.92 & 87.74 & 84.81 & 85.06 & 85.62 & 86.88 & 87.84 \\
\hline
\end{tabular}

$\operatorname{MSPE}\left(\hat{\boldsymbol{\beta}}^{\text {ppm }}\right)$ and $\operatorname{MSPE}\left(\hat{\boldsymbol{\beta}}^{\text {pm }}\right)$ under $\boldsymbol{\beta}=\left\{\frac{j}{100}\right\}_{j=-49}^{j=49}$ (a diffuse signal) when $\boldsymbol{W} \mid \boldsymbol{X} \sim N_{p}\left(\psi \mathbf{1}_{p}+\nu \boldsymbol{X}^{2}, \tau^{2} \boldsymbol{I}_{p}\right)$, where $\boldsymbol{X}^{2}$ indicates the element-wise square of $\boldsymbol{X}$. When $\rho=0.15$, the error structure for $\boldsymbol{X}$ is compound symmetric, and when $\rho=0.75$, the error structure is AR(1). The column-wise minima of each box are in bold 


\begin{tabular}{|c|c|c|c|c|c|c|c|c|c|c|c|}
\hline \multirow[b]{2}{*}{$\rho, R^{2}$} & \multirow[b]{2}{*}{ Method } & \multicolumn{5}{|c|}{$\operatorname{MSPE}\left(\hat{\boldsymbol{\beta}}^{\mathrm{ppm}}\right)$} & \multicolumn{5}{|c|}{$\operatorname{MSPE}\left(\hat{\boldsymbol{\beta}}^{\mathrm{pm}}\right)$} \\
\hline & & $\tau=0.01$ & 0.5 & 1.0 & 1.50 & 2.00 & 0.01 & 0.5 & 1.0 & 1.50 & 2.00 \\
\hline \multirow[t]{6}{*}{$0.15,0.1$} & RIDG & 684.4 & 691.3 & 688.6 & 688.9 & 682.7 & 684.4 & 691.3 & 688.6 & 688.9 & 682.7 \\
\hline & VANILLA & 797.9 & 836.7 & 916.3 & 879.1 & 820.2 & 798.0 & 840.7 & 915.8 & 873.7 & 811.9 \\
\hline & HIERBETAS & 637.6 & 732.2 & 863.1 & 845.8 & 797.4 & 637.6 & 731.7 & 861.2 & 842.7 & 793.4 \\
\hline & EBBETAS & 636.7 & 731.8 & 863.5 & 845.5 & 797.4 & 636.7 & 731.3 & 861.7 & 842.7 & 793.8 \\
\hline & EBSIGMAX & 798.0 & 878.1 & 1085.8 & 1386.5 & 1489.9 & 798.0 & 905.5 & 1223.6 & 1579.1 & 1672.9 \\
\hline & ЕВвотн & 636.7 & 639.3 & 640.4 & 643.2 & 642.0 & 636.7 & 638.9 & 639.2 & 641.5 & 640.0 \\
\hline \multirow{6}{*}{$0.75,0.1$} & RIDG & 307.9 & 305.4 & 304.3 & 309.4 & 310.9 & 307.9 & 305.4 & 304.3 & 309.4 & 310.9 \\
\hline & VANILLA & 350.3 & 410.8 & 486.4 & 451.2 & 404.6 & 350.3 & 408.7 & 477.3 & 438.7 & 390.1 \\
\hline & HIERBETAS & 280.6 & 388.9 & 489.6 & 460.1 & 415.2 & 280.6 & 388.2 & 487.7 & 457.3 & 411.2 \\
\hline & EBBETAS & 280.2 & 388.7 & 490.0 & 460.3 & 415.5 & 280.2 & 388.1 & 488.3 & 457.8 & 411.8 \\
\hline & EBSIGMAX & 350.4 & 366.7 & 384.1 & 420.0 & 455.7 & 350.3 & 379.4 & 420.9 & 487.6 & 536.5 \\
\hline & ЕВВОтн & 280.3 & 280.6 & 283.6 & 287.8 & 292.3 & 280.3 & 280.4 & 283.2 & 287.1 & 291.3 \\
\hline \multirow[t]{6}{*}{$0.15,0.4 \quad 400$} & RIDG & 121.2 & 122.2 & 120.7 & 121.5 & 120.3 & 121.2 & 122.2 & 120.7 & 121.5 & 120.3 \\
\hline & VANILLA & 133.7 & 211.1 & 320.9 & 289.7 & 238.4 & 133.7 & 210.4 & 313.2 & 276.3 & 220.7 \\
\hline & HIERBETAS & 111.6 & 200.4 & 326.9 & 303.0 & 254.2 & 111.6 & 199.8 & 324.3 & 299.0 & 248.7 \\
\hline & EBBETAS & 111.5 & 200.2 & 327.0 & 303.4 & 254.5 & 111.5 & 199.6 & 324.5 & 299.6 & 249.2 \\
\hline & EBSIGMAX & 133.7 & 146.9 & 186.2 & 238.6 & 265.3 & 133.7 & 151.6 & 210.8 & 271.2 & 295.2 \\
\hline & ЕВВОтн & 111.5 & 111.9 & 113.9 & 114.0 & 115.8 & 111.5 & 111.9 & 113.6 & 113.7 & 115.4 \\
\hline \multirow[t]{6}{*}{$0.75,0.4 \quad 400$} & RIDG & 55.7 & 54.4 & 54.7 & 54.1 & 55.0 & 55.7 & 54.4 & 54.7 & 54.1 & 55.0 \\
\hline & VANILLA & 57.9 & 145.7 & 234.1 & 200.5 & 149.5 & 57.9 & 143.1 & 225.9 & 188.6 & 134.4 \\
\hline & HIERBETAS & 48.9 & 148.7 & 245.0 & 214.0 & 164.7 & 48.9 & 147.8 & 242.6 & 210.3 & 159.5 \\
\hline & EBBETAS & 48.9 & 148.6 & 245.1 & 214.3 & 165.0 & 48.9 & 147.8 & 242.8 & 210.7 & 160.0 \\
\hline & EBSIGMAX & 58.0 & 61.8 & 67.3 & 76.7 & 87.5 & 58.0 & 63.9 & 74.0 & 87.9 & 100.9 \\
\hline & ЕВВотн & 48.9 & 49.6 & 51.1 & 53.5 & 55.9 & 48.9 & 49.6 & 51.1 & 53.4 & 55.7 \\
\hline \multirow{6}{*}{$0.15,0.1 \quad 150$} & RIDG & 683.7 & 677.1 & 682.0 & 686.0 & 683.8 & 683.7 & 677.1 & 682.0 & 686.0 & 683.8 \\
\hline & VANILLA & 1251.9 & 1148.7 & 979.6 & 916.3 & 852.8 & 1251.9 & 1162.5 & 1006.0 & 947.1 & 889.4 \\
\hline & HIERBETAS & 646.0 & 653.0 & 687.2 & 703.2 & 695.7 & 646.0 & 652.7 & 685.2 & 699.0 & 690.2 \\
\hline & EBBETAS & 643.8 & 650.7 & 685.9 & 702.4 & 695.4 & 643.8 & 650.5 & 684.2 & 698.8 & 690.6 \\
\hline & EBSIGMAX & 1251.8 & 1484.9 & 2344.1 & 2196.2 & 2188.1 & 1251.8 & 1586.4 & 2523.2 & 2349.6 & 2335.3 \\
\hline & Еввотн & 643.8 & 645.0 & 644.0 & 645.7 & 649.5 & 643.8 & 644.4 & 642.7 & 644.0 & 647.6 \\
\hline \multirow[t]{6}{*}{$0.75,0.1$} & RIDG & 305.1 & 312.5 & 298.8 & 304.3 & 308.0 & 305.1 & 312.5 & 298.8 & 304.3 & 308.0 \\
\hline & VANILLA & 555.2 & 447.8 & 391.1 & 378.0 & 363.4 & 554.9 & 452.6 & 394.4 & 380.1 & 369.4 \\
\hline & HIERBETAS & 288.5 & 294.6 & 326.1 & 340.2 & 330.5 & 288.5 & 294.4 & 324.1 & 335.8 & 325.0 \\
\hline & EBBETAS & 287.5 & 293.7 & 325.9 & 340.2 & 330.6 & 287.5 & 293.5 & 324.1 & 336.2 & 325.5 \\
\hline & EBSIGMAX & 555.9 & 625.2 & 752.6 & 746.4 & 804.2 & 555.8 & 675.6 & 830.0 & 833.3 & 888.0 \\
\hline & ЕВВОтн & 287.5 & 286.9 & 288.0 & 292.3 & 294.7 & 287.5 & 286.6 & 287.4 & 291.5 & 293.9 \\
\hline \multirow[t]{6}{*}{$0.15,0.4 \quad 150$} & RIDG & 122.3 & 121.3 & 120.7 & 120.6 & 120.2 & 122.3 & 121.3 & 120.7 & 120.6 & 120.2 \\
\hline & VANILLA & 209.2 & 199.8 & 202.0 & 192.3 & 178.9 & 209.1 & 201.7 & 201.1 & 186.9 & 175.3 \\
\hline & HIERBETAS & 115.2 & 121.2 & 155.0 & 168.7 & 156.9 & 115.2 & 121.0 & 152.5 & 163.0 & 149.4 \\
\hline & EBBETAS & 114.7 & 120.8 & 155.0 & 169.1 & 157.3 & 114.7 & 120.7 & 152.6 & 163.8 & 150.2 \\
\hline & EBSIGMAX & 209.2 & 250.1 & 416.9 & 390.9 & 392.3 & 209.2 & 267.3 & 445.2 & 418.5 & 418.8 \\
\hline & ЕВВОтн & 114.7 & 115.1 & 115.8 & 118.0 & 118.7 & 114.7 & 114.9 & 115.5 & 117.6 & 118.3 \\
\hline \multirow[t]{6}{*}{$0.75,0.4 \quad 150$} & RIDG & 54.5 & 55.4 & 54.2 & 55.6 & 55.4 & 54.5 & 55.4 & 54.2 & 55.6 & 55.4 \\
\hline & VANILLA & 90.3 & 81.6 & 91.7 & 91.4 & 84.6 & 90.3 & 81.7 & 85.6 & 81.1 & 75.2 \\
\hline & HIERBETAS & 51.5 & 58.2 & 88.3 & 97.2 & 86.4 & 51.5 & 58.0 & 85.5 & 91.3 & 79.2 \\
\hline & EBBETAS & 51.4 & 58.1 & 88.5 & 97.6 & 86.9 & 51.4 & 57.9 & 85.9 & 92.0 & 79.9 \\
\hline & EBSIGMAX & 90.3 & 108.8 & 133.5 & 143.3 & 151.5 & 90.3 & 117.5 & 146.5 & 157.2 & 165.7 \\
\hline & ЕВвотн & 51.3 & 51.8 & 53.2 & 54.8 & 56.4 & 51.3 & 51.7 & 53.1 & 54.8 & 56.3 \\
\hline
\end{tabular}

$\boldsymbol{X} \mid Z \sim N_{p}\left\{1_{[Z=2]}\left(3 \times \mathbf{1}_{p}\right)-1_{[Z=3]}\left(3 \times \mathbf{1}_{p}\right), \boldsymbol{\Sigma}_{\boldsymbol{X}}\right\}$, where $1_{[\cdot]}$ is the indicator function and $Z \stackrel{\text { iid }}{\sim}$ Unif $\{1,2,3\}$. When $\rho=0.15$, the error structure for $\boldsymbol{X}$ is compound symmetric, and when $\rho=0.75$, the error structure is $A R(1)$. The column-wise minima of each box are in bold 


\begin{tabular}{|c|c|c|c|c|c|c|c|c|c|c|c|c|}
\hline \multirow[b]{2}{*}{$\rho, R^{2}$} & \multirow[b]{2}{*}{$n_{\mathrm{B}}$} & \multirow[b]{2}{*}{ Method } & \multicolumn{5}{|c|}{$\operatorname{MSPE}\left(\hat{\boldsymbol{\beta}}^{\mathrm{ppm}}\right)$} & \multicolumn{5}{|c|}{$\operatorname{MSPE}\left(\hat{\boldsymbol{\beta}}^{\mathrm{pm}}\right)$} \\
\hline & & & $\tau=0.01$ & 0.5 & 1.0 & 1.50 & 2.00 & 0.01 & 0.5 & 1.0 & 1.50 & 2.00 \\
\hline \multirow[t]{6}{*}{$0.15,0.1$} & 400 & RIDG & 73.46 & 73.31 & 73.75 & 74.23 & 74.42 & 73.46 & 73.31 & 73.75 & 74.23 & 74.42 \\
\hline & & VANILLA & 79.76 & 75.11 & 72.62 & 72.22 & 72.34 & 79.76 & 75.66 & 73.60 & 73.41 & 73.73 \\
\hline & & HIERBETAS & 67.39 & 67.81 & 68.30 & 68.50 & 68.94 & 67.40 & 67.82 & 68.31 & 68.51 & 68.95 \\
\hline & & EBBETAS & 68.37 & 68.55 & 68.63 & 68.68 & 69.08 & 68.37 & 68.55 & 68.63 & 68.69 & 69.08 \\
\hline & & EBSIGMAX & 79.77 & 88.60 & 109.87 & 142.72 & 152.09 & 79.77 & 91.55 & 124.17 & 161.67 & 171.01 \\
\hline & & ЕВВОТн & 68.37 & 68.54 & 68.72 & 68.74 & 69.11 & 68.37 & 68.54 & 68.71 & 68.73 & 69.11 \\
\hline \multirow[t]{6}{*}{$0.75,0.1$} & 400 & RIDG & 555.96 & 538.87 & 549.68 & 537.04 & 538.92 & 555.96 & 538.87 & 549.68 & 537.04 & 538.92 \\
\hline & & VANILLA & 588.83 & 522.00 & 501.32 & 498.25 & 496.15 & 588.80 & 525.02 & 505.62 & 503.76 & 503.82 \\
\hline & & HIERBETAS & 473.82 & 473.39 & 475.77 & 481.26 & 484.05 & 473.81 & 473.41 & 475.84 & 481.38 & 484.26 \\
\hline & & EBBETAS & 489.08 & 486.78 & 487.50 & 491.78 & 494.03 & 489.07 & 486.79 & 487.53 & 491.85 & 494.16 \\
\hline & & EBSIGMAX & 588.88 & 616.25 & 655.13 & 699.15 & 742.19 & 588.90 & 637.58 & 721.43 & 810.62 & 875.12 \\
\hline & & ЕВВОтн & 489.06 & 485.92 & 485.76 & 489.52 & 494.94 & 489.05 & 485.85 & 485.56 & 489.14 & 494.60 \\
\hline \multirow[t]{6}{*}{$0.15,0.4$} & 400 & RIDG & 17.72 & 17.95 & 17.74 & 17.64 & 17.98 & 17.72 & 17.95 & 17.74 & 17.64 & 17.98 \\
\hline & & VANILLA & 13.30 & 13.10 & 13.93 & 14.73 & 15.22 & 13.30 & 13.15 & 13.97 & 14.76 & 15.25 \\
\hline & & HIERBETAS & 12.38 & 13.14 & 14.78 & 15.93 & 16.42 & 12.38 & 13.13 & 14.78 & 15.94 & 16.43 \\
\hline & & EBBETAS & 13.49 & 14.07 & 15.39 & 16.25 & 16.57 & 13.49 & 14.06 & 15.38 & 16.26 & 16.59 \\
\hline & & EBSIGMAX & 13.30 & 15.25 & 21.20 & 28.61 & 31.23 & 13.30 & 15.82 & 24.05 & 31.96 & 34.42 \\
\hline & & ЕВВотН & 13.49 & 13.84 & 14.71 & 15.38 & 15.77 & 13.49 & 13.85 & 14.72 & 15.40 & 15.79 \\
\hline \multirow[t]{6}{*}{$0.75,0.4$} & 400 & RIDG & 103.33 & 102.79 & 102.81 & 103.07 & 103.18 & 103.33 & 102.79 & 102.81 & 103.07 & 103.18 \\
\hline & & VANILLA & 97.90 & 89.02 & 87.13 & 86.54 & 86.60 & 97.89 & 89.38 & 87.28 & 86.66 & 86.89 \\
\hline & & HIERBETAS & 81.34 & 82.88 & 85.71 & 86.74 & 87.74 & 81.34 & 82.86 & 85.60 & 86.50 & 87.39 \\
\hline & & EBBETAS & 81.65 & 83.71 & 86.20 & 87.56 & 88.38 & 81.65 & 83.69 & 86.10 & 87.35 & 88.07 \\
\hline & & EBSIGMAX & 97.90 & 103.81 & 110.51 & 122.40 & 138.66 & 97.89 & 107.49 & 121.92 & 142.08 & 163.16 \\
\hline & & ЕВВотн & 81.65 & 82.09 & 82.75 & 84.90 & 87.14 & 81.65 & 82.06 & 82.71 & 84.96 & 87.41 \\
\hline \multirow[t]{6}{*}{$0.15,0.1$} & 150 & RIDG & 74.64 & 74.37 & 73.49 & 73.54 & 74.09 & 74.64 & 74.37 & 73.49 & 73.54 & 74.09 \\
\hline & & VANILLA & 125.89 & 115.27 & 96.88 & 89.44 & 86.67 & 125.85 & 116.70 & 100.11 & 93.76 & 91.57 \\
\hline & & HIERBETAS & 68.63 & 68.71 & 68.90 & 69.12 & 69.05 & 68.63 & 68.72 & 68.90 & 69.12 & 69.06 \\
\hline & & EBBETAS & 68.83 & 68.94 & 69.07 & 69.19 & 69.10 & 68.83 & 68.94 & 69.07 & 69.19 & 69.10 \\
\hline & & EBSIGMAX & 125.91 & 152.16 & 241.47 & 238.10 & 228.09 & 125.91 & 162.64 & 258.98 & 252.56 & 242.79 \\
\hline & & ЕВВОTH & 68.83 & 69.00 & 69.16 & 69.30 & 69.30 & 68.82 & 68.99 & 69.16 & 69.28 & 69.28 \\
\hline \multirow[t]{6}{*}{$0.75,0.1$} & 150 & RIDG & 534.33 & 542.65 & 537.26 & 541.66 & 537.41 & 534.33 & 542.65 & 537.26 & 541.66 & 537.41 \\
\hline & & VANILLA & 915.80 & 732.87 & 612.52 & 578.99 & 567.38 & 915.80 & 741.96 & 632.87 & 606.28 & 599.87 \\
\hline & & HIERBETAS & 484.74 & 485.32 & 488.42 & 490.18 & 496.19 & 484.70 & 485.30 & 488.46 & 490.36 & 496.46 \\
\hline & & EBBETAS & 493.54 & 493.14 & 494.38 & 496.59 & 501.44 & 493.53 & 493.11 & 494.39 & 496.67 & 501.54 \\
\hline & & EBSIGMAX & 916.68 & 1039.01 & 1291.71 & 1302.14 & 1285.98 & 916.22 & 1122.00 & 1418.76 & 1436.86 & 1428.32 \\
\hline & & ЕВВОTH & 493.48 & 493.56 & 494.79 & 497.85 & 504.15 & 493.49 & 493.41 & 494.51 & 497.57 & 503.86 \\
\hline \multirow{6}{*}{\multicolumn{2}{|c|}{$0.15,0.4$}} & RIDG & 17.80 & 17.82 & 17.63 & 17.69 & 17.71 & 17.80 & 17.82 & 17.63 & 17.69 & 17.71 \\
\hline & & VANILLA & 20.65 & 20.08 & 18.66 & 18.18 & 18.41 & 20.65 & 20.31 & 19.23 & 18.92 & 19.27 \\
\hline & & HIERBETAS & 13.97 & 14.66 & 15.80 & 16.53 & 16.87 & 13.97 & 14.67 & 15.81 & 16.54 & 16.87 \\
\hline & & EBBETAS & 14.81 & 15.22 & 16.16 & 16.69 & 16.99 & 14.81 & 15.22 & 16.17 & 16.70 & 17.00 \\
\hline & & EBSIGMAX & 20.65 & 26.69 & 45.81 & 45.73 & 46.57 & 20.65 & 28.58 & 48.66 & 48.73 & 49.29 \\
\hline & & ЕВBOTH & 14.81 & 15.18 & 15.80 & 16.15 & 16.46 & 14.81 & 15.19 & 15.81 & 16.16 & 16.47 \\
\hline \multirow{6}{*}{\multicolumn{2}{|c|}{$0.75,0.4$}} & RIDG & 101.28 & 102.88 & 101.80 & 102.30 & 101.53 & 101.28 & 102.88 & 101.80 & 102.30 & 101.53 \\
\hline & & VANILLA & 153.45 & 123.36 & 103.77 & 99.22 & 97.75 & 153.40 & 124.85 & 107.05 & 103.61 & 102.75 \\
\hline & & HIERBETAS & 85.16 & 85.43 & 86.27 & 88.20 & 91.69 & 85.15 & 85.43 & 86.28 & 88.19 & 91.70 \\
\hline & & EBBETAS & 85.13 & 86.21 & 86.54 & 88.70 & 92.03 & 85.13 & 86.21 & 86.56 & 88.71 & 92.09 \\
\hline & & EBSIGMAX & 153.41 & 172.61 & 222.89 & 230.27 & 241.10 & 153.39 & 186.91 & 245.66 & 254.59 & 265.44 \\
\hline & & ЕВВОТН & 85.14 & 86.33 & 86.02 & 88.62 & 89.86 & 85.13 & 86.26 & 85.98 & 88.82 & 90.34 \\
\hline
\end{tabular}

$\boldsymbol{X} \mid Z \sim N_{p}\left\{1_{[Z=2]}\left(3 \times \mathbf{1}_{p}\right)-1_{[Z=3]}\left(3 \times \mathbf{1}_{p}\right), \boldsymbol{\Sigma}_{\boldsymbol{X}}\right\}$, where $1_{[\cdot]}$ is the indicator function and $Z \stackrel{\text { iid }}{\sim}$ Unif $\{1,2,3\}$. When $\rho=0.15$, the error structure for $\boldsymbol{X}$ is compound symmetric, and when $\rho=0.75$, the error structure is AR(1). The column-wise minima of each box are in bold 


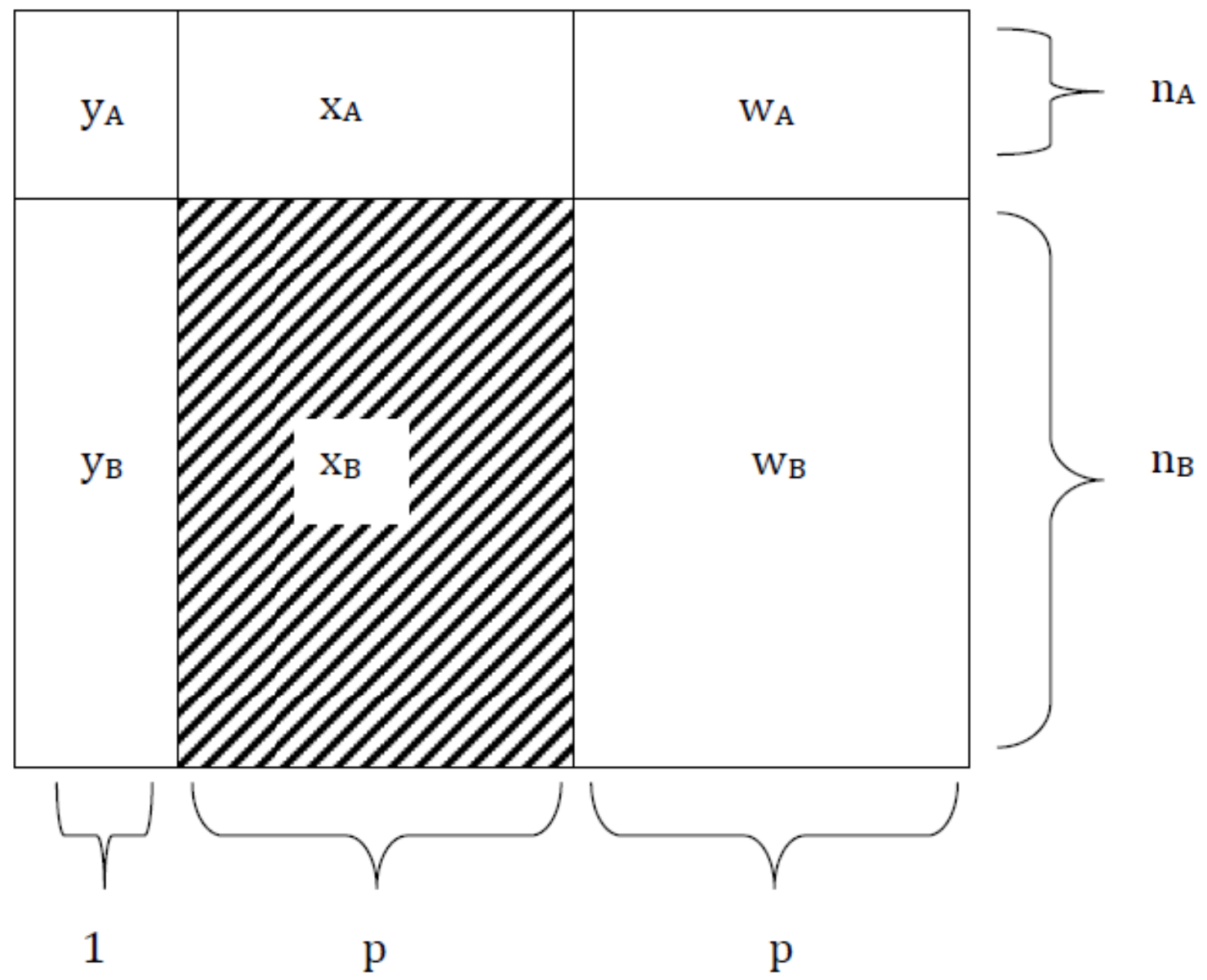

FIG S1. Schematic representation of the prediction problem: $\left(\boldsymbol{y}_{A}, \boldsymbol{x}_{A}, \boldsymbol{w}_{A}\right)$ is measured on $n_{A}$ subjects and $\left(\boldsymbol{y}_{B}, \boldsymbol{w}_{B}\right)$ is measured on $n_{B}$ subjects. $\boldsymbol{x}_{B}$ is considered missing. 